\title{
Platelets exploit fibrillar adhesions to assemble fibronectin matrix revealing new force-regulated thrombus remodeling mechanisms
}

\author{
Sebastian Lickert ${ }^{1}$, Kateryna Selcuk ${ }^{1}$, Martin Kenny ${ }^{2}$, Johanna L. Mehl' ${ }^{1}$, Susanna M. Früh ${ }^{3}$, \\ Melanie A. Burkhardt ${ }^{1}$, Jan-Dirk Studt ${ }^{4}$, Ingmar Schoen ${ }^{2 *}$, Viola Vogel ${ }^{1 *}$ \\ ${ }^{1}$ Laboratory of Applied Mechanobiology, Department of Health Sciences and Technology, ETH Zurich, Vladimir- \\ Prelog-Weg 4, 8093 Zurich, Switzerland \\ ${ }^{2}$ Irish Centre for Vascular Biology, School of Pharmacy and Biomolecular Sciences, Royal College of Surgeons \\ in Ireland, 123 St Stephen's Green, Dublin 2, Ireland \\ ${ }^{3}$ Hahn-Schickard, Georges-Koehler-Allee 103, 79110 Freiburg, Germany \& Laboratory for MEMS Applications, IMTEK \\ Department of Microsystems Engineering, University of Freiburg, Georges-Koehler-Allee 103, 79110 Freiburg, Germany \\ ${ }^{4}$ Division of Hematology, University Hospital Zurich, Rämistrasse 100, 8091 Zurich, Switzerland \\ * corresponding authors: viola.vogel@hest.ethz.ch, ingmarschoen@rcsi.ie
}

\section{ABSTRACT}

Upon vascular injury, platelets are crucial for thrombus formation and contraction, but do they directly initiate early tissue repair processes? Using 3D super-resolution microscopy, micropost traction force microscopy, and specific integrin or myosin lla inhibitors, we discovered here that platelets form fibrillar adhesions. They assemble fibronectin nanofibrils using allbß3 (CD41/CD61, GPIIb-IIla) rather than $\alpha 5 \beta 1$ integrins, in contrast to fibroblasts. Highly contractile platelets in contact with thrombus proteins (fibronectin, fibrin) pull fibronectin fibrils along their apical membrane, whereas platelets on basement membrane proteins (collagen IV, laminin) are less contractile generating less stretched planar meshworks beneath themselves. As probed by vinculin-decorated talin unfolding, platelets on fibronectin generate similar traction forces in apical fibrillar adhesions as fibroblasts do. These are novel mechanobiology mechanisms by which platelets spearhead the fibrillogenesis of the first de novo $\mathrm{ECM}$, including its $2 \mathrm{D}$ versus $3 \mathrm{D}$ network architectures depending on their $\mathrm{ECM}$ environment, and thereby pave the way for cell infiltration. 


\section{INTRODUCTION}

When blood vessels get injured, the massive recruitment and activation of platelets culminates in thrombus formation to stem bleeding, whereas the subsequent infiltration of the clot by immune and stromal cells is essential to ultimately restore tissue integrity (Rodrigues et al. 2019). Platelet adhesion receptors initiate interactions with extracellular matrix (ECM) proteins that become exposed by endothelial barrier disruption, while platelet signaling receptors boost platelet aggregation and granule secretion. Both signal types guide the adoption of several different platelet phenotypes at different times and locations in the thrombus (van der Meijden and Heemskerk 2019; Brass, Diamond, and Stalker 2016). The 'pro-hemostatic' secretion of von Willebrand Factor (VWF), fibrinogen (Fg), and fibronectin (Fn) occurs predominantly at injury site boundaries and in the extravascular portion of the plug (Tomaiuolo et al. 2019). Fibrin(ogen)-binding platelets adopt an aggregating phenotype, while the 'procoagulant' phosphatidylserine (PS) exposing platelets aid the activation of clotting factors (Schoenwaelder et al. 2009) that in turn catalyze the conversion of fibrinogen to fibrin (Fb) and fibrin crosslinking. This enables the build-up of a complex and anisotropic clot architecture (Brass, Diamond, and Stalker 2016). While the molecular regulation of individual components of the hemostatic system has been studied in detail, how to integrate the many fine-tuned platelet-responses at the systems level to ultimately initiate tissue healing is poorly understood, especially with regards to the role played by the rapidly changing extracellular microenvironment (Brass, Diamond, and Stalker 2016; Tomaiuolo et al. 2019).

Studying the response of platelets to free-floating ECM proteins in solution, where these lack their material properties as interconnected networks, has long hampered the elucidation of platelet mechanobiology (Hansen et al. 2018). Platelets contract rapidly when they get in contact with immobilized fibrinogen and reach maximal forces within 15 minutes (Hansen et al. 2018). Actomyosin-based contractility mediates the probing and sensing of substrate stiffness, which modulates platelet activation (Hansen et al. 2018; Zhang et al. 2018) and drives the bundling and alignment of fibrin fibers (Ono et al. 2008) and thus, clot compaction. Contractile platelets then expel the non-contractile, non-adhesive procoagulant platelets to the thrombus surface (Nechipurenko et al. 2019). Graded platelet contractility is thus key to orchestrate fibrin clot remodeling and the local packing density of platelets within the developing thrombus (Brass, Diamond, and Stalker 2016). The inverse question, whether and if so, how platelet contractility is regulated by the microenvironment inside the thrombus or at injury site boundaries in contact with basement membrane debris, is not well understood because a direct comparison of platelet traction forces on different ECM proteins is missing. This question is clinically most relevant because defects in platelet constituents that mediate contractility (adhesion receptors, actin cytoskeleton, myosin Ila) result in compromised 
thrombus formation, thrombus instability, and bleeding (Ono et al. 2008; Ting et al. 2019; Myers et al. 2017; Nurden and Nurden 2008).

After its crucial role to stop bleeding is fulfilled, the fibrin clot has to be gradually replaced with newly assembled ECM fibrils (Burkhardt et al. 2016). It is neither known how this is accomplished without compromising its mechanical stability, nor how the composition and architecture of the thrombus regulate its infiltration by surrounding or homed-in cells. Even the question whether platelets not only secrete ECM proteins, but also contribute to the first steps of tissue repair through assembly of the first provisional Fn matrix is controversial. While plasma fibronectin ( $\mathrm{pFn}$ ) contributes to hemostasis and affects thrombus structure (Wang et al. 2014), and platelet-deposited Fn is deoxycholate-insoluble (Olorundare et al. 2001; J. Cho and Mosher 2006b, 2006a) which suggests that it is in its fibrillar form, the spatial resolution of confocal microscopy falls short to confirm the dimensions of Fn fibrils, while few electron microscopy data are available that suggest that fibrils might be as thin as $20 \mathrm{~nm}$ (Olorundare et al. 2001). At first glance surprising is also why many different ECM protein coatings support Fn deposition by platelets, including Fn and fibrin (Olorundare et al. 2001), laminin (J. Cho and Mosher 2006b), or collagen type I (Jaehyung Cho and Mosher 2006), whereas others such as vitronectin or fibrinogen (Jaehyung Cho et al. 2005) or von Willebrand factor (vWF) (Jaehyung Cho and Mosher 2006) do not. Specific inhibitors against integrins allb $\beta 3$ or $\alpha 5 \beta 1$ reduced Fn deposition on Fn or fibrin substrates to different degrees (Olorundare et al. 2001), but it remained again unclear whether this reduction was due to reduced platelet-substrate adhesion or to reduced Fn fibrillogenesis. Even for fibroblasts, the molecular mechanism how their mechanobiology drives the formation of fibrillar adhesions (Geiger et al. 2001) is still debated and refined (Lu et al. 2020), and nothing is known about the molecular machinery that drives Fn fibrillogenesis in platelets.

Here we employed direct stochastic optical reconstruction microscopy (dSTORM) to resolve the 3D ultrastructure of platelet-matrix adhesion sites, and micropost arrays to quantify how the adhesion site ultrastructure correlates with platelet traction forces exerted on different ECM protein coatings. We discover that platelets can form fibrillar adhesions too, determine conditions under which they form and the integrins involved. We show that platelets assemble the first provisional Fn matrix with fibers that form an in-plane network when platelets are exposed to proteins, while they assemble a 3D Fn network when exposed to blood clot proteins. These findings point to so far unrecognized mechanisms how sensing and responding to their microenvironment is controlled by platelet contractility and we suggest that this contributes to the emergence of the structural heterogeneity of a thrombus. Our findings also challenge the common notion that the infiltration of fibroblasts or mesenchymal stem cells (Rodrigues et al. 2019) is directed by the fibrin scaffold and that these infiltrating cells are the 
first to assemble a de novo Fn ECM (Singh, Carraher, and Schwarzbauer 2010; Barker and Engler 2017).

\section{RESULTS}

\section{Substrate adherent platelets assemble fibronectin nanofibrils.}

Since confocal microscopy falls short to characterize platelet-assembled Fn fibrils (Olorundare et al. 2001; J. Cho and Mosher 2006b, 2006a), we used super-resolution (3D dSTORM) microscopy to analyze washed human platelets on Fn-coated glass and supplemented the medium with fluorescently labeled human pFn that is harvested by cells for fibrillogenesis (G. Baneyx, Baugh, and Vogel 2002) (Figure 1a). Fn fibrils were formed the platelet periphery mainly at two ends (Figure $1 \mathrm{~b}$ and Supplementary Figure S1). Fibrils shorter than one micron were difficult to distinguish unambiguously from deposited aggregates and were thus not investigated further. A statistical analysis of longer fibrils (Supplementary Figure S2) revealed that platelets from different normal healthy donors (Supplementary Figure S3) assembled fibrils with consistent characteristic dimensions. Fn fibrils were few microns long $(L=1.7 \pm 0.43 \mu \mathrm{m}$; Figure 1e) and between $20 \ldots 200 \mathrm{~nm}$ thick $(D=85 \pm 34 \mathrm{~nm}$; Figure 1f). Fibrils were anchored outside of the platelet at the coverslip surface and stretched straight upwards over the platelet lamellipodium (Figure $1 \mathrm{~b}-\mathrm{d}$ ) with a mean start-to-end height difference of $H=243 \pm 123 \mathrm{~nm}$ and an inclination of $\alpha=8.9 \pm 4.9^{\circ}$ (Figure 1g,h). Using the same experimental approach, we see that fibroblasts on Fn (Figure 1i,j) produced longer and thicker Fn fibers due to profilament bundling (Früh et al. 2015) (Figure 1k) but showed the same start-to-end height (Figure 11).

\section{Platelet Fibronectin fibrils align with tensed and polarized actin fiber bundles.}

As Fn fibrillogenesis is initiated by tensile forces that partially expose cryptic Fn-Fn assembly sites (Hocking, Smith, and McKeown-Longo 1996; Gretchen Baneyx and Vogel 1999) and an impaired cytoskeleton reduces Fn deposition by platelets (J. Cho and Mosher 2006b) and by other cells (G. Baneyx, Baugh, and Vogel 2002), we next analyzed the spatial organization of the actin cytoskeleton using dual color (2C) dSTORM. Confirming previous observations (Lickert et al. 2018), we found that the majority of platelets on Fn coatings had formed a highly aligned 'bipolar' filamentous (F-)actin network with pronounced bundles traversing the cell (Figure $1 \mathrm{~m}$ and Supplementary Figure S1). Longer Fn fibrils emanated parallel to these actin cables at adhesion sites situated at both ends and extended beyond the platelet edge (Figure $1 n)$. 
bioRxiv preprint doi: https://doi.org/10.1101/2020.04.20.050708; this version posted April 20, 2020. The copyright holder for this preprint

(which was not certified by peer review) is the author/funder, who has granted bioRxiv a license to display the preprint in perpetuity. It is made available under aCC-BY-ND 4.0 International license.

a) seeding buffer containing $\mathrm{pFn} 647$
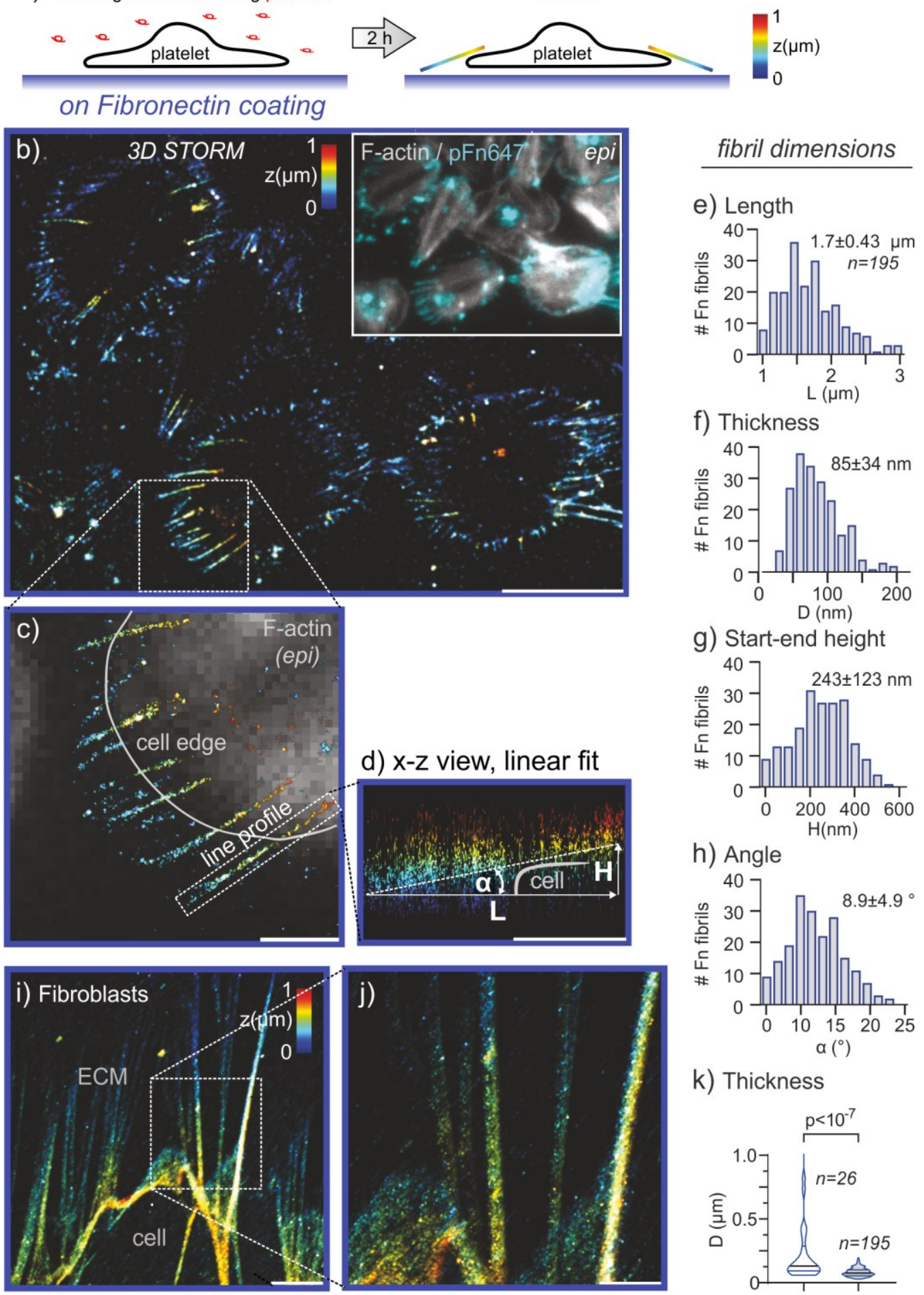

f) Thickness

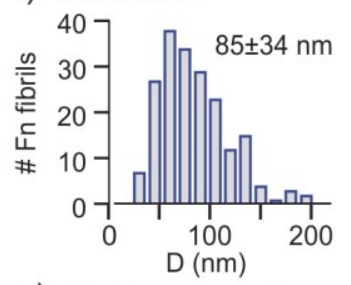

g) Start-end height
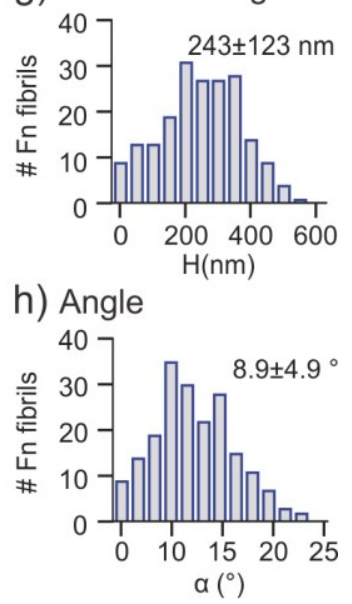

k) Thickness
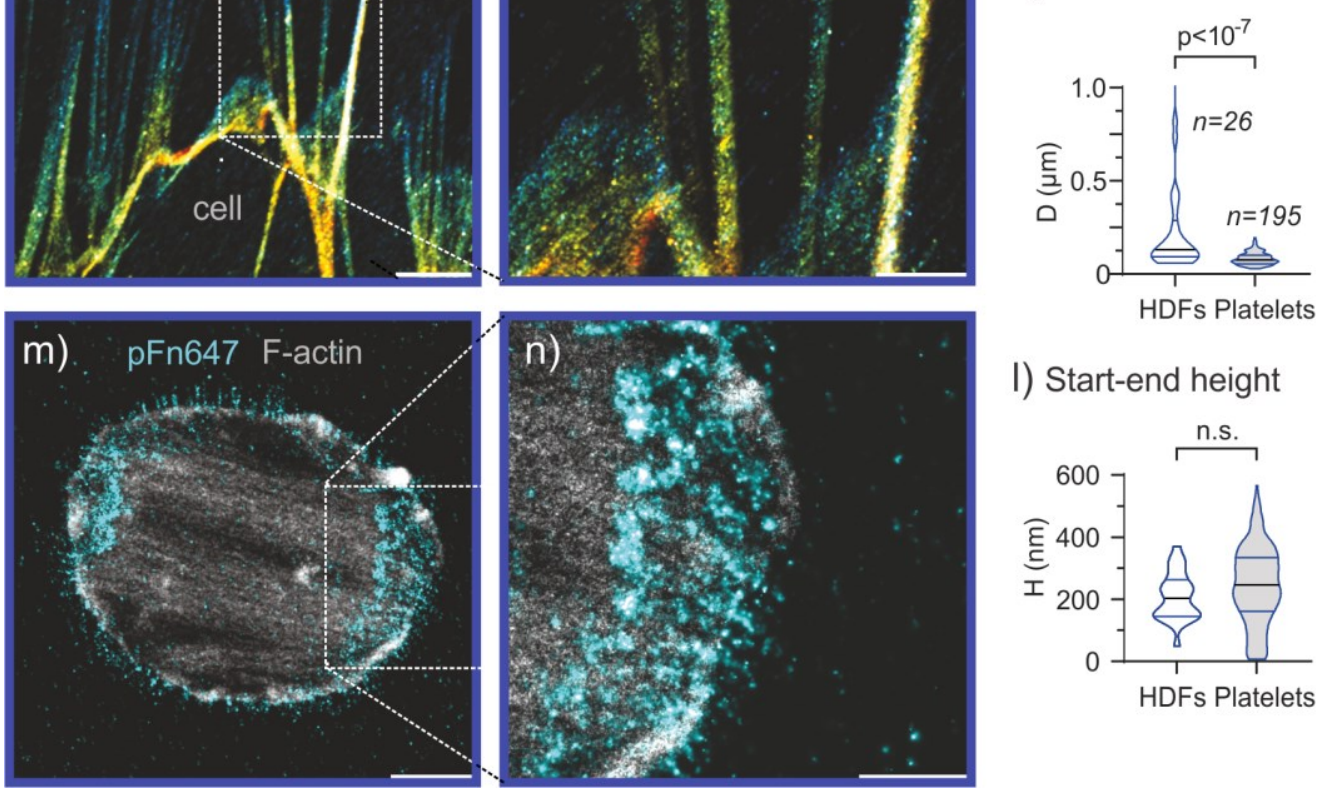

I) Start-end height

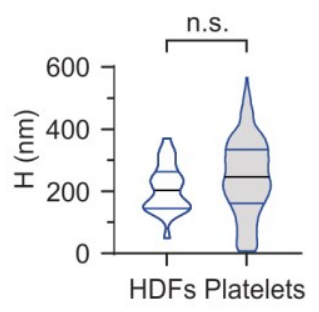


Figure 1. Fibronectin extracellular matrix assembled by human platelets versus by fibroblasts seeded on fibronectin-coated coverslips. (a) Sketch of the experimental procedure. Platelets are seeded on fibronectin

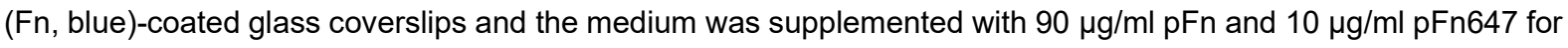
2 hours at $37^{\circ} \mathrm{C}$. The platelets incorporated pFn647 during this time period into their newly assembled Fn fibers. The samples were then fixed for super-resolution imaging. (b) pFn647 imaged by 3D dSTORM. The z-position is color-coded from blue (basal) to red (apical). Scale bar $5 \mu \mathrm{m}$. Inset: epifluorescence (epi) image of F-actin (grey) and pFn (cyan) of the same cells. (c) Magnification of the boxed region of Fn fibrils at the cell edge in (b). Scale bar $1 \mu \mathrm{m}$. (d) $\mathrm{x}-\mathrm{z}$ side view of a single Fn fibril (see boxed region in $\mathrm{c}$ ). The dashed line depicts the linear fit of the localizations of this fibril and its resulting length $(L)$, the start-to-end height $(H)$, and the angle $(\alpha)$ towards the coverslip. Scale bar $1 \mu \mathrm{m}$. (e-h) Dimensions of $195 \mathrm{Fn}$ fibrils extracted from 3D dSTORM images. Data were pooled from five different healthy donors (31-35 years). Only fibrils longer than $1 \mu \mathrm{m}$ were included in the analysis. (i) 3D dSTORM image of Fn-fibrils assembled by human foreskin fibroblasts (HFFs). Scale bar $5 \mu \mathrm{m}$. (j) Magnification of the boxed region of Fn fibrils at the cell edge in (i). Scale bar $2 \mu \mathrm{m}$. Comparison of (k) thickness D and (I) start-end height $\mathrm{H}$ between platelets and fibroblasts. Data distributions are depicted as violin plots showing the median (thick black line) and interquartile ranges (thin colored lines). Data were compared with an unpaired two-tailed MannWhitney test. Adjusted p-values below 0.001 were accepted as highly significant. n.s.: not significant. (m) Representative dual color (2C) dSTORM image of a single platelet spread on Fn with supplemented pFn (cyan) during seeding and stained in addition for F-actin (gray). Scale bar $2 \mu \mathrm{m}$. (n) Magnification of the boxed region of Fn fibrils at the cell edge in $(\mathrm{m})$. Scale bar $1 \mu \mathrm{m}$.

The linear co-alignment of Fn fibrils with F-actin was similar to fibrillar adhesions in nucleated cells (Zamir et al. 2000). In contrast to focal adhesions which only form on the basal side of fibroblasts in 2D cell culture, fibrillar adhesions in fibroblasts are elongated, fibronectinassociated adhesion structures on either the apical or basal side (Katz et al. 2000). Depending on cell type and time, their composition can differ. By forming fibrillar adhesions, $\alpha 5 \beta 1$ integrins are the main drivers of Fn assembly in fibroblasts, yet $\alpha v \beta 3$ can partially compensate in the absence of $\alpha 5 \beta 1$ (Leiss et al. 2008), and $\alpha \mathrm{llb} \beta 3$ integrins overexpressed in $\mathrm{CHO}$ cells also mediate Fn assembly (Wu 1997). Emerging fibrillar adhesions in fibroblasts contain talin, which binds to the NPxY motif of $\beta$-integrins as well as to actin, while over time, talin is being replaced to variable extents by tensin (Katz et al. 2000) controlled by phosphorylation of the NPxY motif (Legate and Fassler 2009). Very recent evidence suggests the existence of an alternative class of fibrillar adhesions in nucleated cells formed on basement membrane proteins through a mechanism in which an early focal adhesion becomes a sliding fibrillar adhesion that deposits Fn fibrils directly onto the basement membrane (Lu et al. 2020). Here we now show striking morphological similarities between the adhesions fibroblasts and platelets exploit to pull fibronectin fibers, whereby their morphology gave them their name "fibrillar adhesions". In contrast to fibroblasts, we see little tensin in the fibrillar adhesions of platelets that were fixed 2 hours after seeding (Supplementary Fig. S4). Future research has to elucidate the time-evolving molecular composition of fibrillar adhesions in platelets. 

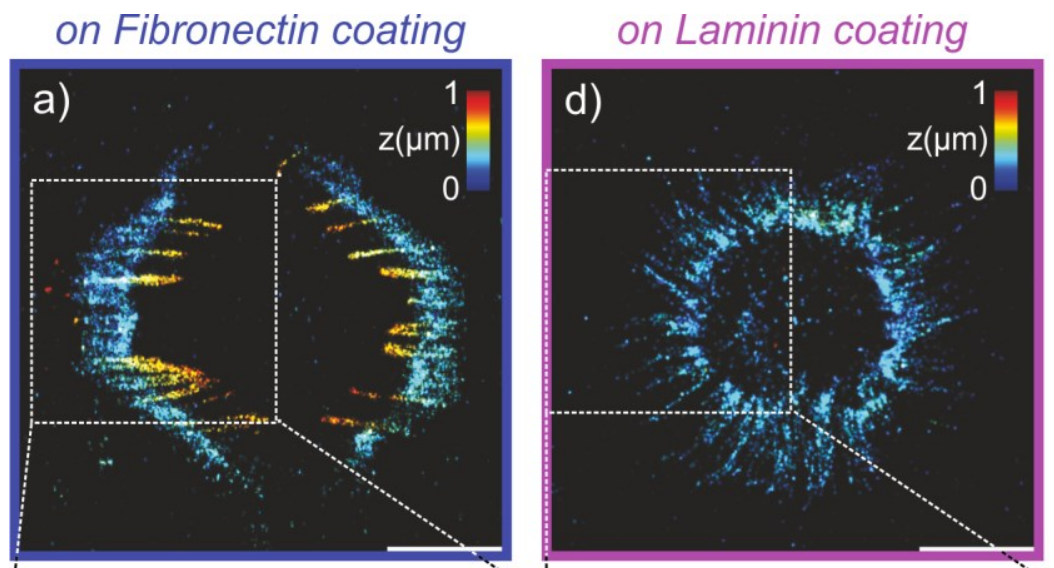

\section{fibril dimensions}
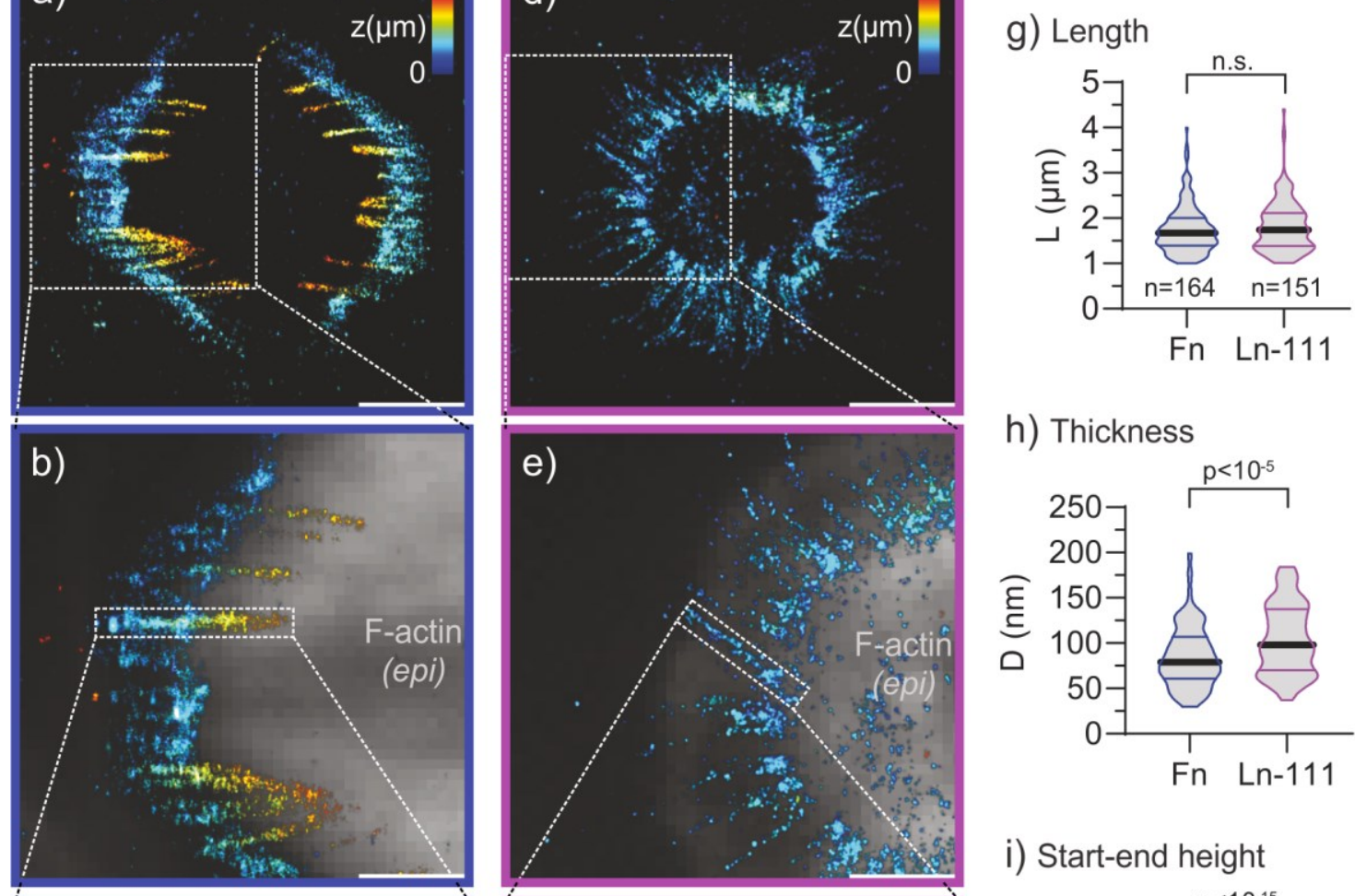

h) Thickness
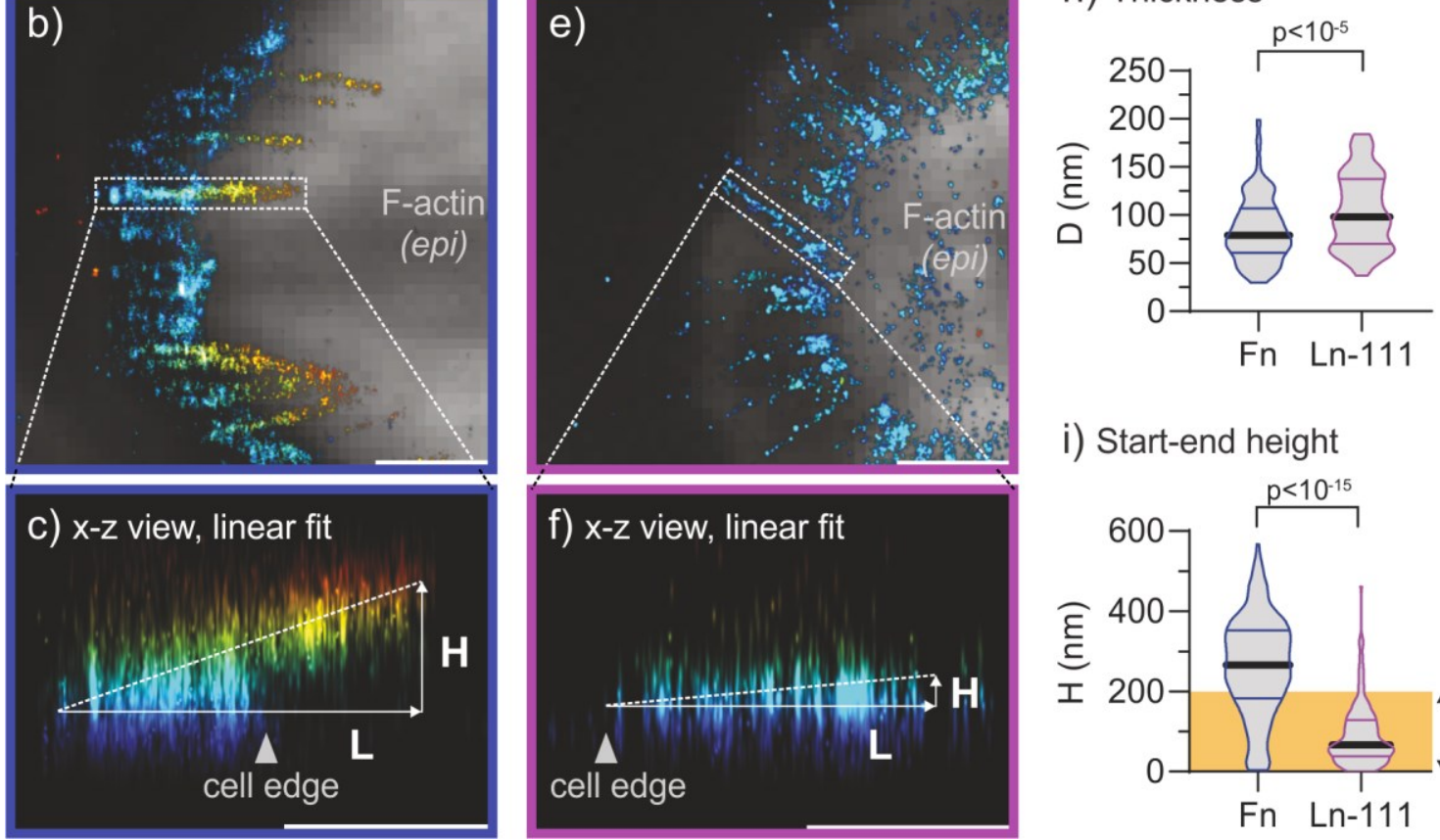

i) Start-end height

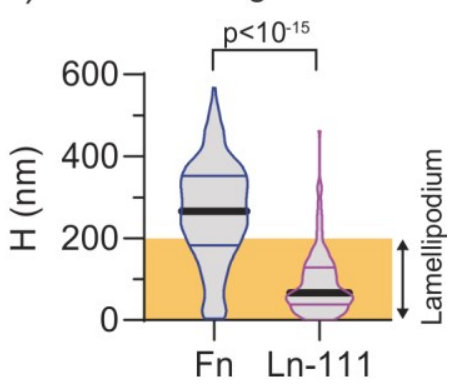

Figure 2. Fibronectin extracellular matrix assembled by human platelets seeded on laminin coatings. See also Supplementary Movie S1. (a) dSTORM image of Fn fibrils assembled in the presence of supplemented pFn647 by a representative platelet spread on Fn. Scale bar $2 \mu \mathrm{m}$. (b) Magnification of the boxed region of Fn fibrils at the cell edge in (a). The dSTORM image is overlaid with the epi image of F-actin (gray). Scale bar $1 \mu \mathrm{m}$. (c) Side view of a single Fn fibril (boxed region in b) and linear fit (dashed line). Scale bar $1 \mu \mathrm{m}$. (d) Fn fibril assembly by a representative platelet spread on a laminin 111 (Ln-111)-coated coverslip. (e) Magnification of the boxed region of Fn fibrils at the cell edge in (d). Note the non-straight appearance of Fn fibrils often observed on Ln-111. (f) Side view of a single Fn fibril (boxed region in e). Scale bar $1 \mu \mathrm{m}$. (g-i) Comparison of Fn fibril dimension on Fn-coatings (blue) and Ln-111 (magenta). The orange box in (i) denotes the height of the platelet lamellipodium (see Supplementary Figure S6). Data were pooled from four healthy human donors (27-35 years); for donor-todonor variation on Fn coatings, see Supplementary Figure S3. Data were compared with an unpaired two-tailed Mann-Whitney test.

\section{Fibronectin fibrils are mostly pulled along the apical membrane of platelets spread on}

\section{fibronectin, while pulled along their basal side on laminin coatings.}

Since platelets sense different ECM proteins after vascular injury, we asked whether the spatial architecture of de novo assembled Fn fibrils depends on the ECM proteins to which the platelets adhere. Fn fibrils were radially oriented on laminin (Ln), in agreement with previously reported Fn deposition patterns (J. Cho and Mosher 2006b), while the actin 
cytoskeleton showed a rather ring-like arrangement (Lickert et al. 2018) (Supplementary Figure S5). In contrast to platelets on Fn that pulled Fn fibrils along their apical side (Figures $2 \mathrm{a}-\mathrm{C}$ ), platelets on laminin assembled $\mathrm{Fn}$ fibrils at their basal side, i.e. between the platelets and the coverslip (Figure $2 d-f$ ). Fibrils on laminin were of similar length $(L=1.8 \pm 0.56 \mu \mathrm{m}$ ) as on Fn and slightly thicker $\left(D=105 \pm 39 \mathrm{~nm}, \mathrm{p}<10^{-5}\right)$, while the start-to-end height $(\mathrm{H}=90 \pm 77 \mathrm{~nm}$, $\left.p<10^{-15}\right)$ and tilt $\left(\alpha=3 \pm 2.84^{\circ}, p<10^{-15}\right)$ were significantly decreased (Figure $2 \mathrm{~g}$-i and Supplementary Figure S5). No statistically significant differences were found for the tested laminin isoforms 111 or 521 (Supplementary Figure S5). We also observed several curvy Fn fibrils on laminin (Figure 2e, arrow) which might indicate that they are less tensed. To validate that most Fn fibrils were attached to the basal platelet membrane, we measured the height of the lamellipodium by imaging the peripheral actin rim at the cell edge and obtained $\sim 200 \mathrm{~nm}$ (Supplementary Figure S6, yellow background in Figure 2i). The fraction of Fn fibrils with $\mathrm{H}$ $>200 \mathrm{~nm}$ was $71 \%$ on Fn coatings, in contrast to $9 \%$ on laminin coatings. To differentiate between these two distinct Fn fibril architectures, we will refer to them as 3D (on Fn) and 2D (on laminin).

allb $\beta 3$ integrins are the sole drivers of fibronectin fibrillogenesis in platelets, not $\alpha 5 \beta 1$ integrins.

Since $\alpha 5 \beta 1$ integrins drive Fn fibrillogenesis in mesenchymal cells, yet the platelet integrin allb $\beta 3$ recognizes Fn's synergy site (Chada, Mather, and Nollert 2006) too, we next asked which integrin was responsible for Fn fibrillogenesis. Both integrins are expressed at very different levels, with a5 $\beta 1$ counts being 2-3\% compared to allb $\beta 3$ (Zeiler, Moser, and Mann 2014). Since inhibition of allb $\beta 3$ or $\alpha 5 \beta 1$ would also interfere with the anchorage to Fn coatings, we conducted the experiments on laminin where platelets adhere independently via a6ß1 (Figure 3a). Blocking allbß3 integrins by the non-priming inhibitor RUC-2 (Zhu et al. 2012) completely abolished Fn fibril formation (Figure 3b). In contrast, blocking $\alpha 5 \beta 1$ by the antibody JBS5 ( $\mathrm{Li}$ et al. 2003) (Figure 3c) showed no differences to the control with regards to the fraction of platelets which assembled Fn fibrils or to fibril length (Figure 3d,e). Note that neither inhibitor affected platelet spreading (Supplementary Figure S7). In contrast to mesenchymal cells, $\alpha 5 \beta 1$ is thus dispensable for Fn assembly by platelets and cannot rescue fibrillogenesis in the absence of $\alpha$ llb $\beta 3$ integrins.

Interestingly, partial inhibition of allbß3 by low dose RUC-2 still allowed normal platelet spreading on Fn coatings (Supplementary Figure S7) yet had a major impact on Fn fibrils which were now pulled along the basal and not along the apical membrane (Figure 3h-j), very similar to laminin coatings. Thus, reducing the density of functional allb $\beta 3$ integrins on the platelet surface switched the dimensionality of the pulled fibrils from $3 D$ to $2 D$. 


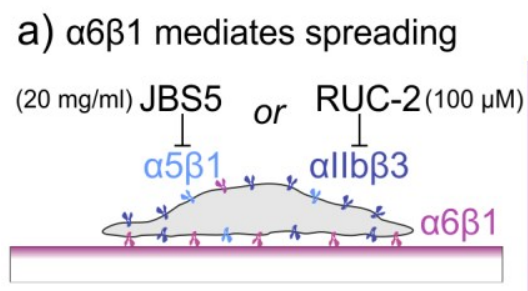

b) allbß3 inhibition (JBS5)
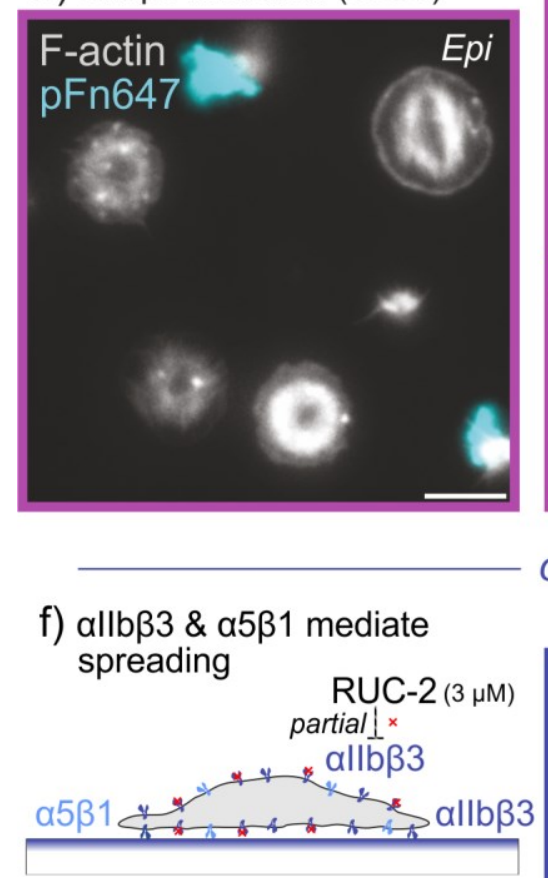

\section{g) Control}

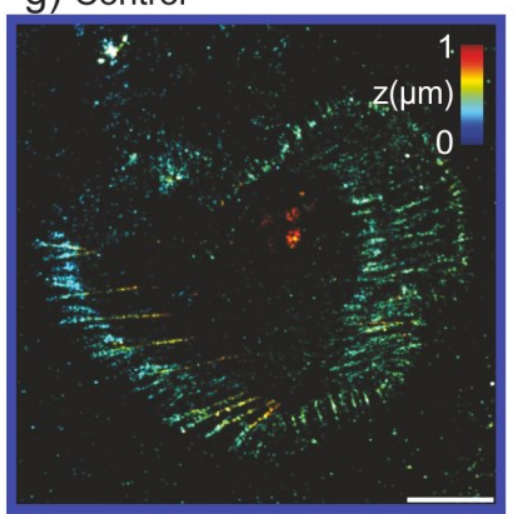

on Laminin coating

c) $\alpha 5 \beta 1$ inhibition (RUC-2)

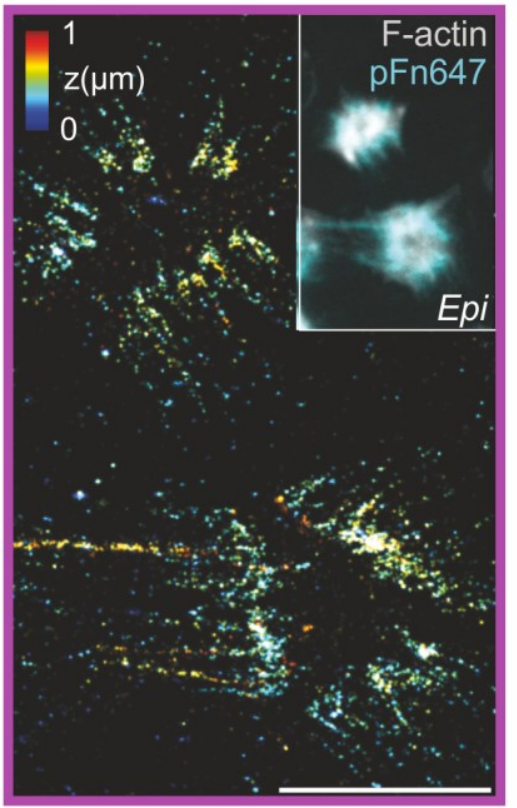

d) Fibril assembly

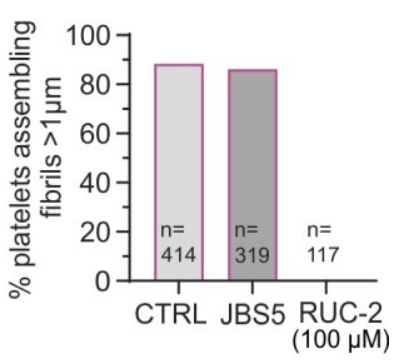

e) Length

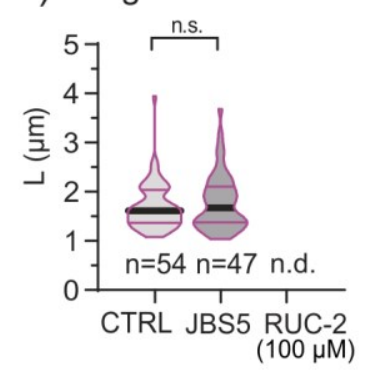

on Fibronectin coating

h) partial allb $\beta 3$ inhibition
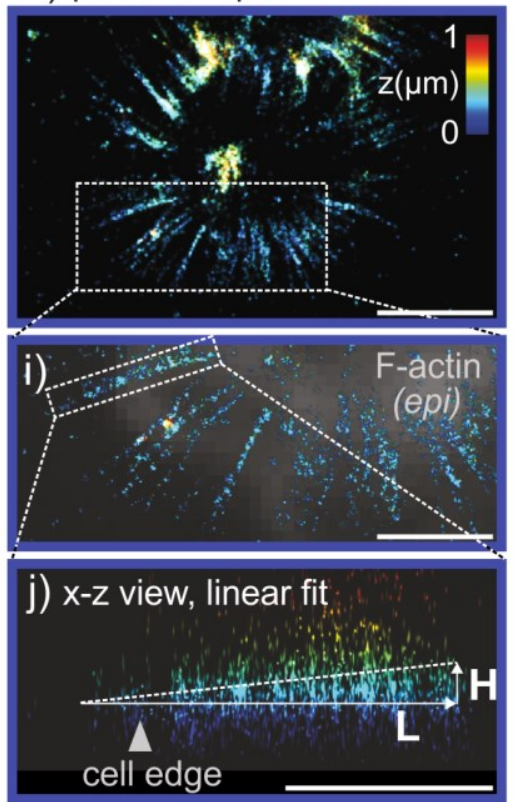

k) Length

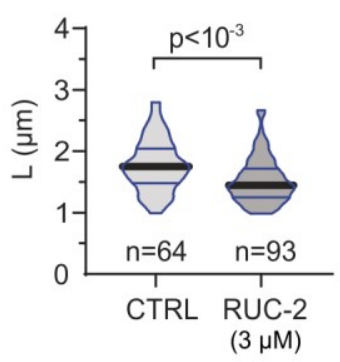

I) Start-end height

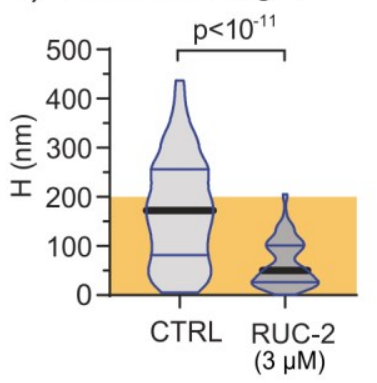

Figure 3. Inhibition of the integrin $\alpha 5 \beta 1$ and allb $\beta 3$ during platelet seeding. (a) Sketch of the experimental approach. A high concentration of either the blocking antibody JBS5 against $\alpha 5 \beta 1(20 \mathrm{mg} / \mathrm{L})$ or of the non-priming inhibitor RUC-2 against allbß3 (100 $\mathrm{MM})$ were supplemented during platelet seeding on Ln-111-coated glass coverslips to block Fn binding through $\alpha 5 \beta 1$ and $\alpha$ llb $\beta 3$, respectively. Laminin allows for platelet spreading through integrin $\alpha 6 \beta 1$ independently of the blocked integrins. (b) Epifluorescence image of F-actin (grey) and pFn647enriched Fn fibrils (cyan) of platelets inhibited with RUC-2 (100 $\mu \mathrm{M})$. Scale bar $5 \mu \mathrm{m}$. (c) 3D dSTORM image of platelets inhibited with JBS5 (20 mg/L). White box: epi image of F-actin (grey) and pFn (cyan) of the same cells. Scale bar $5 \mu \mathrm{m}$. (d) Comparison of the fraction of platelets that produced micron-long fibrils treated with JBS5 and RUC-2, respectively. Epifluorescence images with the indicated number of cells were analyzed. (e) Comparison of Fn fibril length for platelets treated with JBS5 and RUC-2, respectively. Data were obtained from two healthy donors (31 and 33 years). Note that no fibrils were detected for RUC-2 (n.d.: not detectable). (f) Sketch of the experimental approach. A sub-saturating concentration $(3 \mu \mathrm{M})$ of the non-priming allbß3 inhibitor RUC-2 was supplemented 
during platelet seeding to partially block Fn binding of allbß3 on Fn-coated glass coverslips. (g) 3D dSTORM image of platelet assembled Fn fibrils in the presence of pFn647 under control conditions. Scale bar $2 \mu \mathrm{m}$. (h) 3D dSTORM image of Fn fibrils assembled in the presence of $3 \mu \mathrm{M}$ RUC-2. Scale bar $2 \mu \mathrm{m}$. (i) Magnification of the boxed region in (h). The 3D dSTORM image is overlaid with the epi image of F-actin (gray). Scale bar $1 \mu \mathrm{m}$. (j) Side view of a single Fn fibril (boxed region in i). (k-l) Comparison of fibril dimensions. Data were obtained from one male healthy donor (31 years) treated with RUC-2. Data were compared with an unpaired two-tailed MannWhitney test.

\section{Platelet contractility is significantly higher on fibronectin than on laminin coatings.}

We next asked how the observed phenomena relate to platelet contractility. We thus measured traction forces generated by single platelets using micropost arrays that were coated with Fn or laminin by microcontact printing and then passivated to restrict adhesion of platelets to the functionalized post tops (Figure 4a). Platelets spread similarly on either coating and bent posts towards the center of the cell (Figure $4 b-d$ ). A quantification of post deflections yielded higher tractions on Fn compared to laminin, with $2.5 \pm 1.0 \mathrm{nN}$ versus $1.9 \pm 1.1 \mathrm{nN}$ mean force per post, or $18.7 \pm 9.4 \mathrm{nN}$ versus $15.7 \pm 9.4 \mathrm{nN}$ per cell, respectively (Figure $4 \mathrm{e}$ ). The total force per cell as distributed over many posts on Fn was comparable to individual platelets pulling at two anchoring points, i.e. at a suspended AFM tip and a fibrinogen-coated substrate (Lam et al. 2011) or two nearby fibrinogen dots on a compliant polyacrylamide hydrogel (Myers et al. 2017).

\section{Elevated platelet traction forces are required to induce 3D fibronectin fibril assembly.}

To test whether a modulation of contractility was sufficient to explain the differences in Fn fibrillogenesis, we used blebbistatin (BBT) which inhibits human myosin Ila with an $\mathrm{IC}_{50}$ of $5.1 \mu \mathrm{M}$ (Limouze et al. 2004). Blocking contractility with high dose BBT (20 $\mu \mathrm{M})$ led to lessened F-actin bundling and nearly completely abolished the formation of Fn fibrils (Figure 4f). BBT concentrations between $0.3 \mu \mathrm{M}$ and $10 \mu \mathrm{M}$ dose-dependently reduced the fraction of fibrilforming platelets from $80 \%$ to $20 \%$ (Figure $4 \mathrm{~g}$ ), but hardly affected fibril diameter and length (Supplementary Figure S8). Interestingly, fibrils were still anchored at the apical platelet membrane, irrespective of BBT dose (Figure $4 \mathrm{~h}$ ). $3 \mu \mathrm{M}$ and $10 \mu \mathrm{M}$ BBT significantly reduced mean platelet traction forces but still showed a minor fraction of platelets with elevated contractility (Figure 4i). Hypothesizing that these contractile platelets were responsible for the residual Fn fibril assembly, we used the measured fraction of fibril-forming platelets at 0,3 , and $10 \mu \mathrm{M}$ BBT (Figure $4 \mathrm{~g}$ ) and derived a corresponding minimum threshold force from the measured distribution of traction forces, with approximately $0.8 \mathrm{nN}, 0.9 \mathrm{nN}$, and $1.3 \mathrm{nN}$, respectively (Figure 4i). The good agreement suggests that a minimum traction force of $\sim 1 \mathrm{nN}$ per cell-substrate adhesion sites is necessary to induce Fn fibrillogenesis in $3 \mathrm{D}$, and that 
bioRxiv preprint doi: https://doi.org/10.1101/2020.04.20.050708; this version posted April 20, 2020. The copyright holder for this preprint (which was not certified by peer review) is the author/funder, who has granted bioRxiv a license to display the preprint in perpetuity. It is made available under aCC-BY-ND 4.0 International license.

myosin inhibition affects Fn fibrillogenesis in single platelets in an all-or-none fashion, in contrast to the gradual effect of adhesion receptor inhibition (cf. Figure 3h-I).

a) Traction force measurement platform

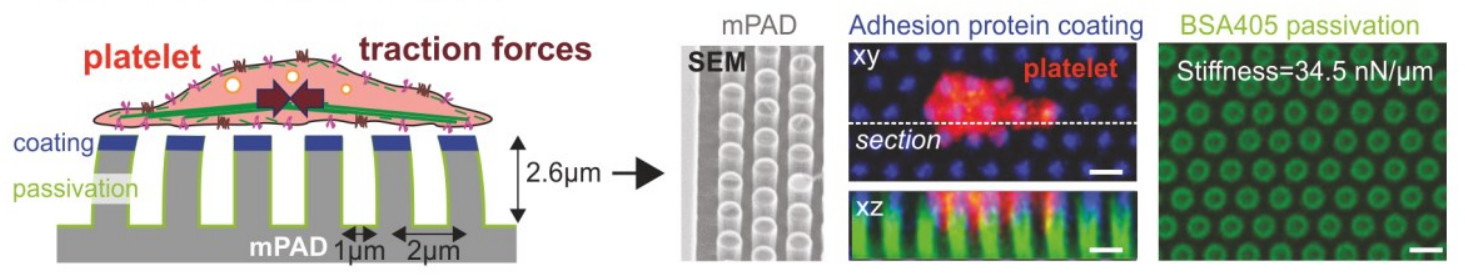

on Fibronectin coating

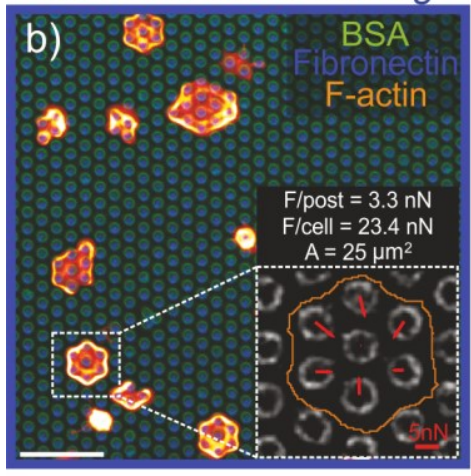

on Fibronectin coating

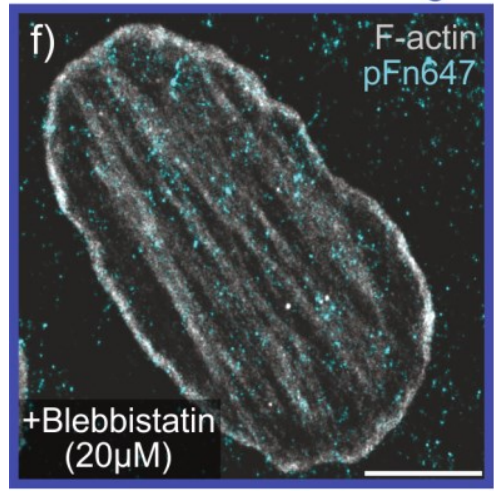

on Laminin coating
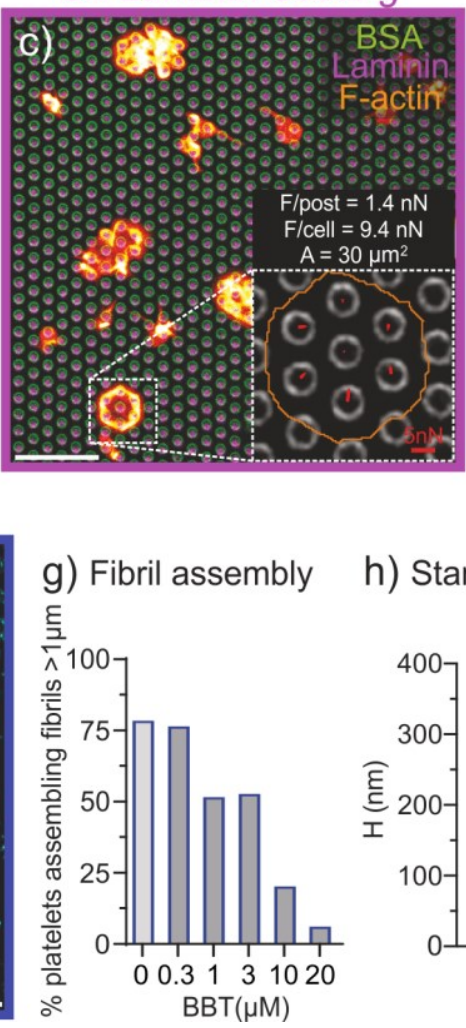

d) Spreading area

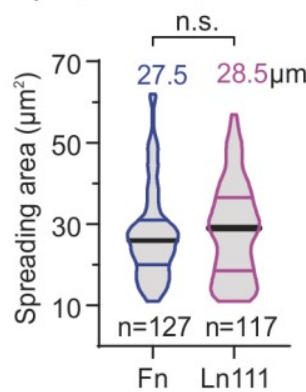

e) Traction forces

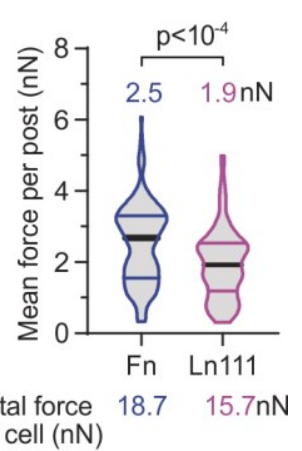

Figure 4. Platelet contractility measured with micropost array detectors (mPADs). (a) Traction forces of single platelets are measured with an optimized micropost array. The tops of the posts are stamped with the respective proteins (blue) and the side walls are passivated (green). Post deflections are determined from the centroids of posts in confocal slices using fluorescently labeled BSA (green). (b,c) Platelets are seeded for one hour on the posts arrays on (b) Fn or (c) Ln-111 and stained for F-actin (red-yellow). Scale bar $5 \mu \mathrm{m}$. Insets: force distribution (red arrows) and measured values of a representative platelet (white boxed region). Scale bars $5 \mathrm{nN}$. (d,e) Comparisons between Fn and Ln-111 in terms of (d) spreading area on posts or of (e) the mean force per post and total force per platelet. Platelets were obtained from a healthy male donor (33 years) and data were compared with an unpaired two-tailed Mann-Whitney test. (f) Representative 2C dSTORM image of a platelet seeded on Fn in the presence of the myosin inhibitor blebbistatin (BBT, $20 \mu \mathrm{M}$ ). As Fn fibril assembly is mostly stalled, pFn647 is seen as cyan dots. Scale bar $2 \mu \mathrm{m}$. (g-i) Dose dependent effects of BBT on (g) the fraction of platelets that produced micron-long fibrils, (h) fibril start-end height $\mathrm{H}$ and (i) mean force per post. The red line in (i) indicates the contractile force level that platelets have to overcome to form $\mathrm{Fn}$ fibrils as derived from these experiments. Platelets were obtained from two healthy male donors (31 and 33 years) and data were compared with a non-parametric KruskalWallis rank test with post-hoc Dunn test. 


\section{The mechanomolecular strain in fibrillar adhesions is the same in fibroblasts and platelets on fibronectin coatings.}

To determine whether the different forces in cell-substrate adhesions also result in different forces in fibrillar adhesions, we used 2C dSTORM to characterize the molecular build-up of the latter. In fibroblasts, fibrillar adhesions are formed as Fn-bound integrins are coupled via talin to actin fibers which are pulled via myosin II towards the cell center inducing fibrillogenesis along the pulling direction (Geiger et al. 2001). Thereby, talin gets stretched and partially unfolds, opening up binding sites for vinculin which re-enforces these molecular linkages during adhesion maturation (Atherton et al. 2016; Huang et al. 2017). Talin unfolding is accompanied by an increasing spatial offset between integrins and vinculin (Case et al. 2015; Liu et al. 2015). In platelets spread on Fn, vinculin was strongly localized to peripheral adhesion sites and formed string-like patterns that were co-aligned with F-actin bundles (Lickert et al. 2018). Fn fibrils overlapped with these elongated vinculin patterns and were tightly registered (Figure 5a,b), similar to fibrillar adhesions in fibroblasts (Zamir et al. 2000). Analysis of the localization density along linear vinculin-Fn patterns (Supplementary Figure S9), where vinculin decorates stretched talin, revealed a mean spatial offset of $381 \pm 195 \mathrm{~nm}$ between the terminal ends of Fn fibrils and vinculin patches $(n=60$; Figure $5 g, h)$. A similar mean offset of $375 \pm 278 \mathrm{~nm}$ is seen for fibroblasts on Fn coatings (Figure 5e,f,h). This suggests that the mechanical strain of the integrin-talin-vinculin-actin connections that are part of force-bearing apical fibrillar adhesion sites in platelets and apical fibrillar adhesion sites in fibroblasts are similar. On laminin coatings, the offset between vinculin and Fn was only $157 \pm 90 \mathrm{~nm}(\mathrm{n}=64$; Figure $5 \mathrm{c}, \mathrm{d}, \mathrm{h})$ and thus significantly smaller. The observed offset range is consistent with measured force-induced talin rod extensions of $60 \ldots 350 \mathrm{~nm}$ (Margadant et al. 2011 ) and the measured shallow orientation $\sim 15^{\circ}$ of talin within adhesions (Liu et al. 2015).

\section{The dimensionality of the fibronectin fibril networks is different whether platelets adhere to adhesion proteins from blood clots versus basement membranes.}

We finally asked whether our findings regarding Fn assembly could be generalized to specific thrombus sites. Besides Fn, we selected fibrin as the major clot component since spreading on fibrin but not fibrinogen supports Fn matrix assembly by platelets (Jaehyung Cho et al. 2005). Platelets on fibrin coatings showed the typical allbß3-dependent contractile phenotype (Lickert et al. 2018) and formed Fn fibrils mainly localized in the periphery of the cell (Figure $6 a)$. As on Fn coatings, Fn fibrils on fibrin were mainly pulled along the apical membrane (Figure 6c) and only a moderate increase in fibril length and thickness were noted (Supplementary Figure S8). Besides laminin, collagen type IV (Col4) is the most abundant component of the vascular basement membrane. Fn fibrils on Col4 coatings (Figure 6b) 
bioRxiv preprint doi: https://doi.org/10.1101/2020.04.20.050708; this version posted April 20, 2020. The copyright holder for this preprint (which was not certified by peer review) is the author/funder, who has granted bioRxiv a license to display the preprint in perpetuity. It is made available under aCC-BY-ND 4.0 International license.

recapitulated the architecture on laminin with respect to their anchorage beneath the cell (Figure 6c), length and thickness (Supplementary Figure S8).
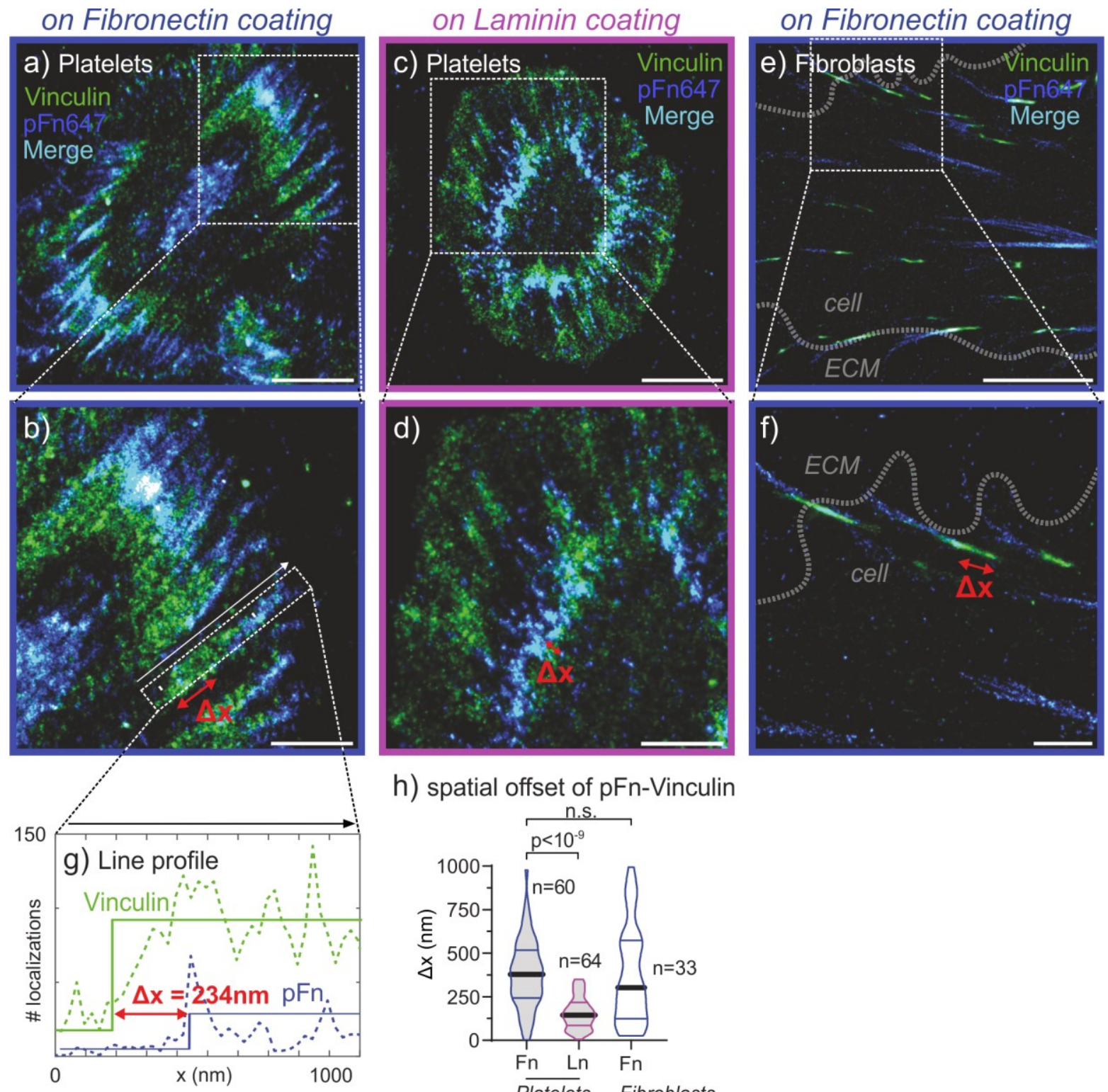

h) spatial offset of pFn-Vinculin

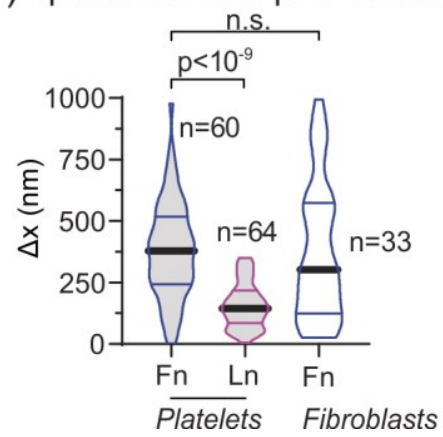

Figure 5. Dual-color dSTORM of fibrillar adhesions in platelets and fibroblasts. (a) A representative platelet spread on a Fn coating with medium supplemented pFn (blue) that gets incorporated into the platelet-assembled Fn fibers. The samples were then fixed and stained in addition for vinculin (green). Scale bar $2 \mu \mathrm{m}$. (b) Magnification of the boxed region of Fn fibrils at the cell edge in (a). Scale bar $1 \mu \mathrm{m}$. Red arrow denotes the spatial offset of the vinculin stain with respect to the $\mathrm{Fn}$ fibril. (c,d) A representative platelet spread on Ln-111. Representation as in (a,b). (e,f) A representative fibroblast seeded on Fn. Scale bars: (e) $5 \mu \mathrm{m}$, (f) $1 \mu \mathrm{m}$. (g) Exemplary line profiles (dashed) of the localization density along the line in (b) for the vinculin stain (green) and the pFn stain (blue). Each profile was fitted with a step function to obtain the spatial offset between stains. (h) Comparison of the spatial offset between Fn fibrils and vinculin for platelets on Fn and Ln-111 and for fibroblasts on Fn. Platelets on Fn and Ln were obtained from two same donors, a healthy male and a female donor (30 years and 27 years). Data were compared with a non-parametric Kruskal-Wallis rank test with post Dunn test to make (multiple) comparisons. 
bioRxiv preprint doi: https://doi.org/10.1101/2020.04.20.050708; this version posted April 20, 2020. The copyright holder for this preprint (which was not certified by peer review) is the author/funder, who has granted bioRxiv a license to display the preprint in perpetuity. It is made available under aCC-BY-ND 4.0 International license.
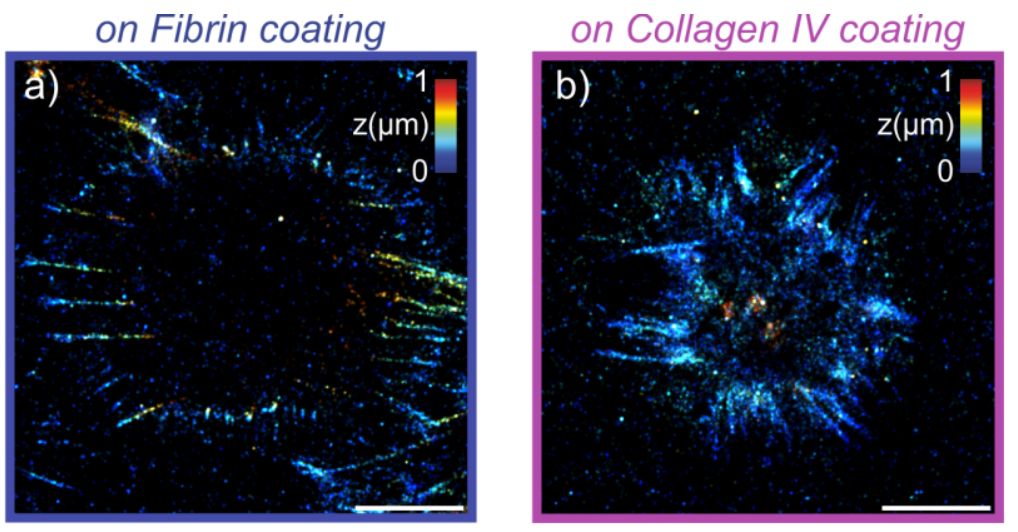

C) Start-end height

d)

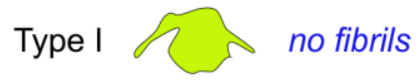

Type II

"2D-like"

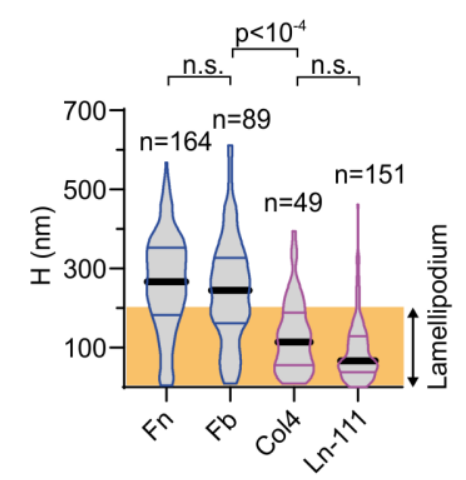

\section{Low}

Platelet contractility

Type III 1 "3D-like"
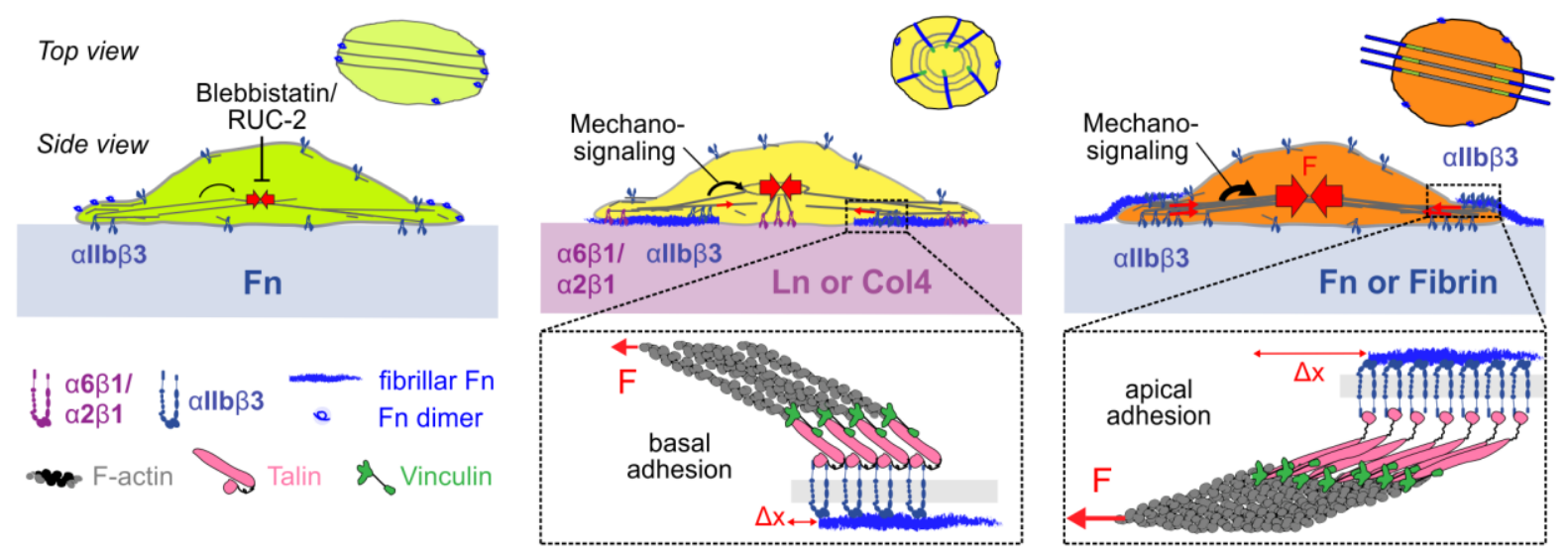

e)

Initial Platelet Deposition

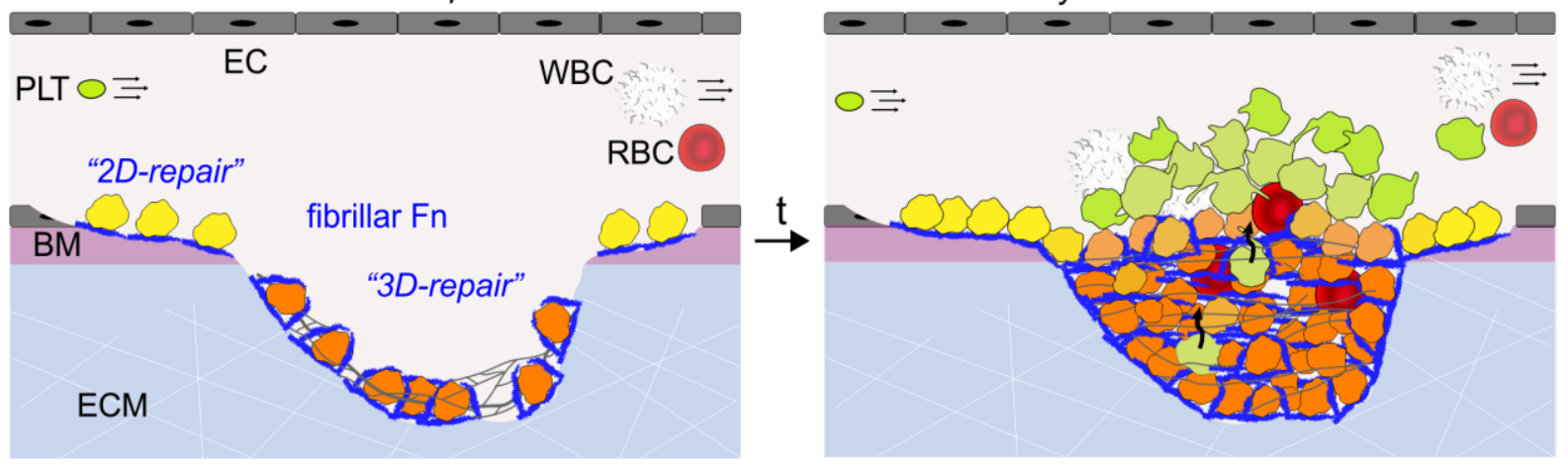

Figure 6. Fibronectin assembly of platelets on adhesion proteins of the provisional clot and the basement membrane. (a) 3D dSTORM image of pFn647-enriched Fn fibril assembly by a single platelet seeded on fibrin (Fb, binds allb $\beta 3$ ). See also Supplementary Movie S1. Scale bar $2 \mu \mathrm{m}$. (b) 3D dSTORM image of pFn647-enriched Fn fibril assembly by a single platelet seeded on collagen type IV (Col-4, binds $\alpha 2 \beta 1)$. Scale bar $2 \mu \mathrm{m}$. (c) Systematic comparison of fibril start-end height between Fn (blue), Fb (blue), Col-4 (magenta) and Ln-111 (magenta). Data were pooled in addition to Figure 2 from two healthy human donors (31-33 years) and were compared with a non-parametric Kruskal-Wallis rank test with post-hoc Dunn test to make (multiple) comparisons. (d) Integrin mechanosensing of ECM proteins triggers dimensionality of the first deposited ECM network. Platelets spread on $\mathrm{Fn}$ and $\mathrm{Fb}$ (right) generate high traction forces (red arrows and dark red-yellow) and Fn fibrils are aligned to polarized actin bundles (top view). Mechanosignaling (black arrow) through allbß3 (blue) instructs Fn fibril anchorage along the apical membrane of platelets and the mechanomolecular strain induces a spatial offset between vinculin (green) and Fn (see inset). On Ln or Col4 (middle), platelets contract less strong (yellow) and 
form radial oriented $\mathrm{Fn}$ fibrils (top view) pulled beneath their basal side with a small spatial offset between vinculin and Fn (side view, inset). Dose-dependent inhibition of myosin Ila and the allbß3 (left) further reduces platelet contractility (green) and prevents Fn fibril formation. (e) Based on these three phenotypes, we propose a model for the initial thrombus formation under flow. After rupture of the vessel wall, platelet adhesion to different ECM proteins tunes platelet contractility and orchestrates a distinct repair process. Platelet deposition on basement membrane (BM) proteins leads to a "2D-like" repair process with a less stretched planar Fn network. Platelets in contact with clot proteins (Fn and Fb) strongly contract and assemble a "3D-like" Fn network under high tension in the core of the clot and push less contractile platelets towards the thrombus surface. As a result, the fibrin network in the clot gets interlaced with Fn fibrils.

\section{DISCUSSION}

How the local multifactorial biochemical and mechanical cues are sensed by platelets and direct their response to allow for a spatially well-organized repair of injured vessel walls versus scaring while maintaining mechanical integrity remains elusive. We discovered here by superresolution microscopy that platelets can form fibrillar adhesions as fibroblasts do (Figures 1,5), but that they exploit allbß3 rather than $\alpha 5 \beta 1$ integrins to drive Fn fibrillogenesis (Figures 3). For fibroblasts it is well established that mechanotransduction processes require the cooperation of mechano-sensing structural proteins, including talin, vinculin and tensin that connect the contractile cytoskeleton to the integrin tails and get stretched by forces, and of additional signalling molecules whose phosphorylation triggers downstream cell signalling programs, including FAK, Src and paxillin (Kechagia, Ivaska, and Roca-Cusachs 2019), yet it was not known that platelets can form fibrillar adhesions too. It is thus remarkable that platelets not only orchestrate thrombus formation and contraction, but also direct the initial tissue repair process by assembling Fn nanofibrils (Figures 1,2,6). This de novo ECM fibrillogenesis by platelets is in line with previous observations made at lower optical resolution on platelets alone (Olorundare et al. 2001; Jaehyung Cho et al. 2005; J. Cho and Mosher 2006a; Jaehyung Cho and Mosher 2006) or in blood clots (Burkhardt et al. 2016), and suggests that fibrillar Fn might play an overlooked role in the formation and remodeling of the hemostatic plug and the guidance of cell infiltration from the surrounding.

Our findings taken together with existing literature can be summarized in a new mechanoregulated thrombus formation model (Figure $6 \mathrm{~d}, \mathrm{e}$ ): we now propose the existence of at least three different platelet phenotypes, defined by either low, medium or high contractility. Each subtype serves contractile-specific functions, including their ability to sense their ECM environment and to respond by either being procoagulant or assembling de novo ECM. These three contractile phenotypes are not directly congruent with the well-known platelet phenotypes defined by $\alpha$-/ס-granule secretion or PS exposure (van der Meijden and Heemskerk 2019). On one hand, more than one biochemical phenotype has low contractility, such as resting but also PS positive platelets (Zhang et al. 2018; Nechipurenko et al. 2019; 
Agbani et al. 2015). On the other hand, little is known about the contractility of platelets that have or have not undergone degranulation. The intricate feedback loops of both, platelet activation and platelet mechanosensing, and the cross-talk between them, demand a more comprehensive characterization of contractility in different platelet phenotypes to refine our proposed model.

Strongly contracting platelets push procoagulant or other non-contractile platelets to the thrombus surface (Nechipurenko et al. 2019) and thereby establish the basic core-shell architecture (Brass, Diamond, and Stalker 2016). Here we find that platelets in contact with proteins of the vessel wall are less contractile than when in contact with clot proteins (Figure 4). Platelet contractility drives the build-up of fibrillar adhesions (Figure 5 ) that coordinate the organization of distinct Fn fibril networks which either encage the platelets when adhering to Fn or fibrin (Figures 1,6) or are pulled beneath them on vascular basement membrane proteins (Figures 2,6). Extrapolated from our findings, the ECM microenvironment tunes platelet contractility at different thrombus locations (Figure 6e) suggesting that the platelets in contact with blood clot proteins play a far more active role in the wound site contraction. The fibrinand Fn-rich extraluminal thrombus portion in penetrating injuries (Tomaiuolo et al. 2019; Wang et al. 2014) locally enhances compaction by maximizing platelet contraction, while platelets of medium contractility mediate the thrombus anchorage at the vascular basement membrane upon superficial injury. How additional chemical stimuli like gradients of platelet agonists or PS exposure could synergize with ECM proteins to up- (Olorundare et al. 2001) or downregulate (Zhang et al. 2018) platelet contractility and Fn fibrillogenesis needs further investigation. $2 \mathrm{D}$ spatial cues might guide invading cells to move along the plane that still contains basement membrane proteins or their fragments thus helping to restore the tissue barrier. The less stretched planar Fn fibril networks might further direct the $2 \mathrm{D}$ healing process by templating the reassembly of collagens (Kubow et al. 2015) for basement membrane repair, as during vascular morphogenesis (Hielscher et al. 2016) or in other tissues (Filla et al. 2017). An extensive Fn network at basement membranes is known to guide cell migration during development (Lu et al. 2020; Duband and Thiery 1982). We thus hypothesize that the plateletdeposited Fn fibrils at basement membranes could serve an analogous role during tissue repair. The progressive development of a tensed 3D network of Fn fibrils in the core might guide infiltrating cells or allow migrating platelets (Gaertner et al. 2017) to reposition themselves within a lesion. Our finding that the force which platelets apply per allb $\beta 3$ integrin to Fn fibrils equals the force which fibroblasts apply to the $\alpha 5 \beta 1-\mathrm{Fn}$ bonds, as probed here by the displacement of vinculin in fibrillar adhesion sites (Figure 5), is fascinating and likely of major physiological importance because it enables platelets to withstand the pulling forces of 
invading fibroblasts and hence ensures that the plug does not lose its mechanical stability during remodeling.

In platelets, $\alpha \mathrm{llb} \beta 3$ and not $\alpha 5 \beta 1$ integrins are the sole drivers of $\mathrm{Fn}$ stretching and fibrillogenesis (Figure 3). Adhesion signaling of either $\alpha 6 \beta 1$ on laminin, $\alpha 2 \beta 1$ on Col4, or allb $\beta 3$ on Fn or fibrin is sufficient to drive $\alpha$ llb $\beta 3$ to assemble Fn fibrils (Figures 1,2,6), in analogy to the pre-activation of $\alpha 5 \beta 1$ integrins by $\alpha v \beta 3$ in fibroblasts (Zamir et al. 2000; Bharadwaj et al. 2017), the initiation of $\alpha 5 \beta 1$-containing sliding fibrillar adhesions by $\alpha 3 \beta 1$ or $\alpha 2 \beta 1$ integrins on Matrigel (Lu et al. 2020), and in accordance with previous observations (Olorundare et al. 2001; Jaehyung Cho et al. 2005; J. Cho and Mosher 2006b, 2006a; Jaehyung Cho and Mosher 2006). Adhesion signaling through allb $\beta 3$ is essential to develop full contractility (Figure 4), maximal stress fibers (Lickert et al. 2018), as well as a 3D apical anchorage of Fn fibrils as seen in fibroblasts (Zamir et al. 2000) (Figure 1) and a similar mechanomolecular strain within fibrillar adhesions (Figure 5). We for the first time derive a threshold of $\sim 1 \mathrm{nN}$ per cell-substrate adhesion to activate allbß3 integrins to form 3D fibrils (Figure 4). The ECM proteins from the vessel wall and the thrombus thus essentially instruct the platelets to adopt distinct phenotypes of medium and high contractility, respectively, probably serving different functions as guided by different levels of mechanosensitive integrin signaling (Strohmeyer et al. 2017; Chen et al. 2019). This mechanism in platelets is, probably due to their small size and the high abundance of $\alpha$ llb $\beta 3$ integrins, different from fibroblasts. Even though $\alpha 5 \beta 1$ integrins compete with allb $\beta 3$ for their common ligand $\mathrm{Fn}$ and drive $\mathrm{Fn}$ fibrillogenesis in fibroblasts (Singh, Carraher, and Schwarzbauer 2010) (Figures 1,5), they surprisingly fail to rescue fibril assembly in platelets (Figure 3 ). The platelet kindlin 3 equally promotes integrin $\beta 1$ and $\beta 3$ clustering (Malinin et al. 2009) and talin binds either integrin, ruling out a deficiency for a specific adaptor protein for $\alpha 5 \beta 1$ in the myeloid lineage (Humphries et al. 2009). With 1'500 copies per cell (Zeiler, Moser, and Mann 2014) and a typical average force of $\sim 5 \mathrm{pN}$ per integrin (Zhang et al. 2018), all a5 $\beta 1$ integrins together could only sustain $7.5 \mathrm{nN}$ over prolonged time. This suggests that $\alpha 5 \beta 1$ integrins on platelets are merely outcompeted by the abundant allbß3 during Fn fibrillogenesis. Although we did observe abundant tensin-1 immunostaining in platelets, in agreement with previous proteomic reports (Sun et al. 2007; Sabrkhany et al. 2018), tensin-1 did not localize to neither focal nor to fibrillar adhesion sites (Supplementary Figure S4). Since both, the NPLY motif of $\beta 3$ integrins (Law, Nannizzi-Alaimo, and Phillips 1996) as well as myosin (Zimman et al. 2014; Solari et al. 2016), get phosphorylated upon thrombin-induced platelet aggregation, which might suggest a potential switch from talin to tensin in the contractile phenotype, further investigations are needed to delineate the functions of tensin-1 and of many other adaptor proteins in the fibrillar adhesions of platelets. 
Beyond discovering that platelets can form fibrillar adhesions, our data reveal major new outside-in mechanisms by which the ECM identity of the microenvironment seen by platelets regulates their contractility, which vice versa adapts their phenotype leading to the built up of a heterogeneous thrombus and paving the way for cell invasion. In response to the ECM identity, which is rapidly changing in a fresh wound site, platelets change their contractility and the dimensionality of the first provisional ECM fibers which they assemble. This suggest new mechanisms by which the ECM microenvironment might direct location-specific responses, which upon integration, steer the early phases of a spatially well-organized tissue repair process. Beyond initiating blood coagulation and contracting blood clots, we now propose that the ability of platelets to interlace Fn fibers into the fibrin clot from early time points throughout its maturation might ensure that the mechanical stability of blood clots is maintained as the first provisional matrix gets degraded and replaced by newly formed tissue. We postulate that any factor that interferes with platelet Fn fibrillogenesis will thus contribute to bleeding disorders for example as resulting from myosin Ila mutations leading to MYH9 diseases (Nurden and Nurden 2008). Our findings have also direct relevance for how blood-biomaterial interactions (Burkhardt et al. 2016) instruct tissue repair processes and will probably guide the future development of wound healing scaffolds.

While our study investigated fundamental mechanisms of fibrillar adhesion formation by using isolated platelets, in order to specify the phenomenon, the integrins involved and how fibronectin fibrillogenesis is regulated by platelet contractility, we can only speculate here how the three platelet phenotypes might impact blood clot formation, contraction and the subsequent invasion of cells. Our mechanistic sketch (Figure 6e) though is in agreement with our previous observations of Fn fibrillogenesis and fibroblast invasion made on 2 and 24 hours old blood clots that had formed on titanium surfaces (Burkhardt et al. 2016). 


\section{METHODS}

\section{Reagents}

Reagents were purchased from Sigma Aldrich, if not mentioned otherwise. Acid citrate dextrose (ACD) tubes (Sol. B, Vacutainer ${ }^{\circledR}, \mathrm{BD}$, Switzerland); coverslips (18 mm diameter, thickness 1.5; Hecht-Assistent, Germany); human fibrinogen (FG; F3879); human fibronectin (FN; purified from plasma as described previously (Früh et al. 2015)); human collagen type IV (Col4; C8374); murine laminin (LN-111; L2020); human laminin (LN-521, Biolaminin521 LN, BioLamina, Sweden); bovine serum albumin (BSA; 05470); Adenosine 5'-diphosphate sodium salt (ADP; A2754); thrombin from human plasma (T6884); RUC-2 (gift from Prof. B.S. Coller, New York University); Blebbistatin (B0560); mouse anti-integrin a5ß1 (MAB1969); mouse antivinculin (V9131); unconjugated donkey anti-mouse or anti-rabbit lgG (Jackson Immunoresearch, USA); Alexa Fluor 647 NHS ester (A20006, ThermoFisher, USA); CF680 NHS ester (92139, Biotium, USA); Alexa Fluor 546 NHS ester (A20002, ThermoFisher, USA); DyLight 405 NHS ester (A46400, ThermoFisher, USA); goat anti-mouse CF680 (SAB4600361); Alexa Fluor 488 Phalloidin (A12379, ThermoFisher, USA); Alexa Fluor 647 Phalloidin (A22287, ThermoFisher, USA); human Factor XIII (Fibrogammin ${ }^{\circledR} 1250$, CSL Behring); Float-A-Lyzer G2 MWCO 20kD dialysis columns (Z726834-12EA); TI Prime (MicroChemicals, Germany); photoresist AZ 1505 (MicroChemicals, Germany); developer AZ 726 MIF (MicroChemicals, Germany); Sylgard 184 Silicone Elastomer (Dow Corning, USA); hard PDMS (PP2-RG07, Gelest, USA); Trichloro $(1 \mathrm{H}, 1 \mathrm{H}, 2 \mathrm{H}, 2 \mathrm{H}$-perfluorooctyl)silane (448931), Pluronic ${ }^{\circledR}$ F-127 (P2443).

\section{Labeling of plasma fibronectin (Fn) and BSA with fluorescent dyes}

Purified $\mathrm{Fn}$ (in $1 \mathrm{M}$ arginine in PBS) was stored at $-80^{\circ} \mathrm{C}$ before use. The random labeling of surface accessible lysine residues of $F n$ was obtained by amid bond formation with fluorescent probes as described previously (Früh et al. 2015). In short, Fn was transferred into an amine labeling buffer (0.1 $\mathrm{M} \mathrm{NaHCO}_{3}$ in PBS, $\left.\mathrm{pH} 8.5\right)$ and incubated with 20-fold molar excess of Alexa Fluor 647 succinimidyl ester for 1 hour at room temperature. Free dye was removed and buffer exchanged to PBS. As measured by absorption, Fn-AF647 (denoted as pFn647) batch carried 10-15 dye molecules per molecule on average. For 2C STORM of pFn and Factin, pFn was labeled with CF680 in an analogue way. For mPAD experiments, BSA was labeled with DyLight 405 and adhesion proteins were labeled with Alexa Fluor 488, resulting in 5-7 dyes per molecule.

\section{Platelets and sample preparation for dSTORM imaging}


Fn, Ln and Col4 were coated with a concentration of $100 \mu \mathrm{g} / \mathrm{ml}$ (in PBS) over night at $4^{\circ} \mathrm{C}$ onto coverslips as described previously (Lickert et al. 2018). Cross-linked fibrin matrix was generated as described before (Jaehyung Cho et al. 2005) by mixing human fibrinogen $(500 \mu \mathrm{g} / \mathrm{mL})$; thrombin (3 unit/mL), FXIII $(5 \mu \mathrm{g} / \mathrm{mL})$, and $\mathrm{CaCl}_{2}(2 \mathrm{mM})$ in $0.5 \mathrm{~mL}$ Tris-buffered saline $(20 \mathrm{mM}$ Tris- $\mathrm{HCl}, \mathrm{pH} \mathrm{7.4}$, and $150 \mathrm{mM} \mathrm{NaCl}$ and incubation of coverslips with this mixture overnight at $4{ }^{\circ} \mathrm{C}$. All coverslips were washed thoroughly 3 times with PBS before use. Ethical approval was obtained from the Kantonale Ethikkommission Zurich (KEK-ZH-Nr. 20120111) and RCSI Research Ethics Committee (REC1391 and REC1504) prior to the commencement of the study. All experiments were performed in accordance with relevant guidelines and regulations. Whole blood from healthy adult volunteers was collected in ACD tubes. Isolated washed platelets were resuspended in Tyrode's buffer (TB) (TB containing 1.8 $\mathrm{mM} \mathrm{Ca}{ }^{2+}, 5 \mu \mathrm{M}$ ADP, $90 \mu \mathrm{g} / \mathrm{ml} 10 \mu \mathrm{g} / \mathrm{ml}$ Fn-AF647, and, where appropriate, RUC-2, Blebbistatin or anti-integrin $\alpha 5 \beta 1$. After seeding on coverslips for 2 hours at $37^{\circ} \mathrm{C}$, platelets were rinsed with TB, fixed with $3 \%(\mathrm{w} / \mathrm{v})$ paraformaldehyde (FA) in TB for 15 minutes and washed with PBS (see also Supplementary Information).

\section{Fabrication, optimization and imaging of the micropost substrates}

Resist-coated silicon wafers were patterned with circles (Diameter: $1 \mu \mathrm{m}$; Center-to-center spacing: $2 \mu \mathrm{m}$ ) using $220 \mathrm{~nm}$ deep UV lithography (ABM, USA). Then, the resists were etched with a fluorine-based inductively coupled plasma (ICP) process (PlasmaPro100 Estrelas, Oxford Instruments, United Kingdom) to create $2.6 \mu \mathrm{m}$ deep post structures. Master structures were replicated by creating elastomeric negative molds using a sandwich of spin-coated hard PDMS and Sylgard 184, and subsequent molding using hard PDMS on glass coverslips. The spring stiffness of posts was calculated as $34.51 \mathrm{nN} / \mu \mathrm{m}$ (Schoen et al. 2010). The top surface of the posts was then coated with adhesion protein (1:1 mixture of labeled/unlabeled) by contact printing after UV/Ozone activation. The remaining accessible mPAD surface was stained and passivated with fluorescent BSA $(0.4 \mathrm{mg} / \mathrm{mL})$ and then $0.5 \%(\mathrm{w} / \mathrm{v})$ Pluronics F127. Washed platelets from healthy volunteers were seeded for $1 \mathrm{hr}$ on the arrays and subsequently fixed with $3 \%(\mathrm{w} / \mathrm{v})$ FA in PBS for 15 minutes. Samples were stained with phalloidin 647 and fluorescent z-stacks were acquired by confocal microscopy (Leica SP8, or Zeiss Examiner Z.1) at a pixel size of $60-70 \mathrm{~nm}$. The deflection of posts was determined from the BSA channel. In short, positions of individual posts were determined by template matching and a radial symmetry fit. Positions of single posts were linked through slices of the z-stack. The lateral offset between slices was determined by a redundant cross-correlation of nondeflected posts in the region around cells and corrected. The deflection profile of posts was approximated by a c-spline, yielding deflection amplitude and directions of post tops. Forces per post were calculated by Hooke's law using the spring stiffness. The median apparent force 
of posts in the region outside of cells was taken as the measurement resolution. The spreading area of cells was determined from outlines based on the F-actin stain, and the mean force per posts and the total force per cell were determined for each cell from the posts beneath it.

\section{dSTORM imaging of fibronectin and the cytoskeleton}

During platelet seeding and spreading, the medium was supplemented with $10 \mu \mathrm{g} / \mathrm{ml}$ of pFn647 and $90 \mu \mathrm{g} / \mathrm{ml}$ unlabeled pFn that get incorporated into the Fn fibrils assembled by platelets, as previously observed for fibroblasts (Baneyx et al. 1999). Afterwards, samples were fixed and stained for the actin cytoskeleton by incubating with Alexa Fluor 488 phalloidin at 1:50 dilution for $1 \mathrm{~h}$ for epifluorescence. For $2 \mathrm{C}$ STORM of $\mathrm{pFn}$ and F-actin, Alexa Fluor 647 phalloidin was used instead. For 2C STORM of pFn and vinculin, samples were incubated overnight at $4^{\circ} \mathrm{C}$ with $1: 60$ dilution of anti-vinculin antibody in $3 \%(w / v)$ BSA. Next, samples were rinsed three times in PBS and incubated for $1 \mathrm{~h}$ with 1:60 dilution of anti-mouse CF680 in 3\% BSA. After 3 washes in PBS, samples were post-fixed with 4\% PFA in PBS for 15 min. A home-built set-up was used for single-molecule localization microscopy, as previously described (Früh et al. 2015). Fitting and analysis of dSTORM movies was performed using the software SMAP (developed by Dr. Jonas Ries, EMBL Heidelberg).

\section{Analysis of the fibril dimensions}

Fn fibrils were analyzed as shown exemplary in Supplementary Figure S2. The dSTORM images were post-processed in SMAP and only z-localizations with a localization precision better than $100 \mathrm{~nm}$ further analyzed. Single fibers were manually marked from start to end by a line $\mathrm{ROI}$ which defined the $\mathrm{x}$-coordinate. The length was taken as the length of the line. The diameter was defined as the full width half maximum (FWHM) of a Gaussian fit of the perpendicular line profile with $2 \mathrm{~nm}$ binning. The inclination and start-to end height were determined by a line fit to $z$-localizations binned into 20 intervals along $x$.

\section{Analysis of the lateral offset between vinculin and Fn fibrils at fibrillar adhesion sites}

Lines were drawn along fibrillar adhesion sites from inside the cell towards to outside anchorage of the Fn fibrils. Fluorophore localizations in both channels (vinculin and Fn) that were within a $150 \mathrm{~nm}$ wide region around these lines were rotated to align them in the $\mathrm{x}$ direction. The start of the vinculin adhesion and of the Fn fibril were set to the 0.05 quantile of $\mathrm{x}$-positions of localizations in the respective channel. This procedure allowed for a robust determination of the signal boundaries in the presence of background localizations (Supplementary Figure S9).

\section{Manual count of Fn fibrils}


Fraction of platelets which assembled Fn were measured manually. At least thirty different field of views $\left(48.1 \times 48.1 \mu^{2}\right)$ were captured in the pFn channel on the epifluorescence microscope and platelets which assemble pFn fibrils (fibril length $>1 \mu \mathrm{m}$ ) and platelets which only deposit $\mathrm{pFn}$ (fibril length $<1 \mu \mathrm{m}$ ) were separately counted and the ratio determined.

\section{Acknowledgements}

We thank Prof. Barry S. Coller for generously providing the RUC compounds and Dr. Jonas Ries (EMBL Heidelberg) for kindly providing software for the visualization of dSTORM data. We would like to acknowledge Lukas Braun for the helpful discussion during the manuscript preparation. This work was supported by ETH Zurich, by Swiss TransMed "Life Matrix" 33/2013 (V.V.), Wyss Zurich (V.V.), the Velux Stiftung (K.S.), as well as by the RCSI (I.S.), and has received funding from the European Union's Horizon 2020 research and innovation programme under grant agreement No 747586 (I.S.).

\section{Author contributions}

S.L., I.S. and V.V. laid out the major concepts of the study; M.A.B. performed initial fibrillogenesis experiments with platelets; S.L. and I.S. planned experiments; S.L., K.S. and M.K. performed all platelet spreading experiments; S.M.F. performed cell culture experiments with fibroblasts; S.L. with help of K.S. and J.L.M. performed super-resolution imaging for platelets, S.M.F. performed super-resolution imaging of fibroblasts; S.L. and I.S. wrote the script for fibril analysis and analyzed all super-resolution data; S.L fabricated the micropost arrays; S.L., M.K. and I.S. optimized the micropost arrays; S.L. and M.K performed, imaged and analyzed platelet traction force experiments; I.S. wrote the script for the traction forces analysis; S.L., I.S. and V.V. wrote the manuscript. All authors critically revised the report.

\section{Competing financial interests}

The authors declare no competing financial interests.

\section{Additional information}

ORCID profiles:

S.L.: 0000-0002-0569-9362

K.S.: 0000-0002-9232-4971

M.K.: 0000-0003-3055-8075

J.L.M.: 0000-0002-6140-1970

I.S.: 0000-0002-5699-1160

V.V.: 0000-0003-2898-7671 


\section{REFERENCES}

Agbani, Ejaife O., Marion T.J. van den Bosch, Ed Brown, Christopher M. Williams, Nadine J.A. Mattheij, Judith M.E.M Cosemans, Peter W. Collins, Johan W.M. Heemskerk, Ingeborg Hers, and Alastair W. Poole. 2015. "Coordinated Membrane Ballooning and Procoagulant Spreading in Human Platelets." Circulation 132 (15): 1414-24. https://doi.org/10.1161/CIRCULATIONAHA.114.015036.

Atherton, Paul, Ben Stutchbury, Devina Jethwa, and Christoph Ballestrem. 2016.

"Mechanosensitive Components of Integrin Adhesions: Role of Vinculin." Experimental Cell Research 343 (1): 21-27. https://doi.org/10.1016/j.yexcr.2015.11.017.

Baneyx, G., L. Baugh, and V. Vogel. 2002. "Fibronectin Extension and Unfolding within Cell Matrix Fibrils Controlled by Cytoskeletal Tension." Proceedings of the National Academy of Sciences 99 (8): 5139-43. https://doi.org/10.1073/pnas.072650799.

Baneyx, Gretchen, and Viola Vogel. 1999. "Self-Assembly of Fibronectin into Fibrillar

Networks underneath Dipalmitoyl Phosphatidylcholine Monolayers: Role of Lipid Matrix and Tensile Forces." Proceedings of the National Academy of Sciences 96 (22): 12518-23. https://doi.org/10.1073/pnas.96.22.12518.

Barker, Thomas H., and Adam J. Engler. 2017. "The Provisional Matrix: Setting the Stage for Tissue Repair Outcomes." Matrix Biology 60-61 (July): 1-4.

https://doi.org/10.1016/j.matbio.2017.04.003.

Bharadwaj, Mitasha, Nico Strohmeyer, Georgina P. Colo, Jonne Helenius, Niko

Beerenwinkel, Herbert B. Schiller, Reinhard Fässler, and Daniel J. Müller. 2017. “AV-

Class Integrins Exert Dual Roles on A5 $\beta 1$ Integrins to Strengthen Adhesion to

Fibronectin." Nature Communications 8 (1): 14348.

https://doi.org/10.1038/ncomms14348.

Brass, Lawrence F., Scott L. Diamond, and Timothy J. Stalker. 2016. "Platelets and Hemostasis: A New Perspective on an Old Subject." Blood Advances 1 (1): 5-9. https://doi.org/10.1182/bloodadvances.2016000059.

Burkhardt, Melanie A, Jasmin Waser, Vincent Milleret, Isabel Gerber, Maximilian Y Emmert, Jasper Foolen, Simon P Hoerstrup, Falko Schlottig, and Viola Vogel. 2016. "Synergistic Interactions of Blood-Borne Immune Cells, Fibroblasts and Extracellular Matrix Drive Repair in an in Vitro Peri-Implant Wound Healing Model." Scientific Reports 6 (1): 21071. https://doi.org/10.1038/srep21071.

Case, Lindsay B, Michelle A Baird, Gleb Shtengel, Sharon L Campbell, Harald F Hess, Michael W Davidson, and Clare M Waterman. 2015. "Molecular Mechanism of Vinculin Activation and Nanoscale Spatial Organization in Focal Adhesions." Nature Cell Biology 17 (7): 880-92. https://doi.org/10.1038/ncb3180. 
Chada, Diwakar, Timothy Mather, and Matthias U. Nollert. 2006. "The Synergy Site of Fibronectin Is Required for Strong Interaction with the Platelet Integrin Allbß3." Annals of Biomedical Engineering 34 (10): 1542-52. https://doi.org/10.1007/s10439-006-91611.

Chen, Yunfeng, Lining Arnold Ju, Fangyuan Zhou, Jiexi Liao, Lingzhou Xue, Qian Peter Su, Dayong Jin, et al. 2019. "An Integrin Allbß3 Intermediate Affinity State Mediates Biomechanical Platelet Aggregation." Nature Materials 18 (7): 760-69. https://doi.org/10.1038/s41563-019-0323-6.

Cho, J., and D. F. Mosher. 2006a. "Role of Fibronectin Assembly in Platelet Thrombus Formation." Journal of Thrombosis and Haemostasis 4 (7): 1461-69. https://doi.org/10.1111/j.1538-7836.2006.01943.x.

Cho, J., and Deane F. Mosher. 2006b. "Characterization of Fibronectin Assembly by Platelets Adherent to Adsorbed Laminin-111." Journal of Thrombosis and Haemostasis 4 (5): 943-51. https://doi.org/10.1111/j.1538-7836.2006.01862.x.

Cho, Jaehyung, Jay L. Degen, Barry S. Coller, and Deane F. Mosher. 2005. "Fibrin but Not Adsorbed Fibrinogen Supports Fibronectin Assembly by Spread Platelets." Journal of Biological Chemistry 280 (42): 35490-98. https://doi.org/10.1074/jbc.M506289200. Cho, Jaehyung, and Deane F Mosher. 2006. "Impact of Fibronectin Assembly on Platelet Thrombus Formation in Response to Type I Collagen and von Willebrand Factor." Blood 108 (7): 2229-36. https://doi.org/10.1182/blood-2006-02-002063.

Duband, Jean Loup, and Jean Paul Thiery. 1982. "Distribution of Fibronectin in the Early Phase of Avian Cephalic Neural Crest Cell Migration." Developmental Biology 93 (2): 308-23. https://doi.org/10.1016/0012-1606(82)90120-8.

Filla, Mark S., Kaylee D. Dimeo, Tiegang Tong, and Donna M. Peters. 2017. "Disruption of Fibronectin Matrix Affects Type IV Collagen, Fibrillin and Laminin Deposition into Extracellular Matrix of Human Trabecular Meshwork (HTM) Cells." Physiology \& Behavior 165: 7-19. https://doi.org/10.1016/j.physbeh.2017.03.040.

Früh, Susanna Maria, Ingmar Schoen, Jonas Ries, and Viola Vogel. 2015. "Molecular Architecture of Native Fibronectin Fibrils." Nature Communications 6: 7275. https://doi.org/10.1038/ncomms8275.

Gaertner, Florian, Zerkah Ahmad, Gerhild Rosenberger, Shuxia Fan, Leo Nicolai, Benjamin Busch, Gökce Yavuz, et al. 2017. "Migrating Platelets Are Mechano-Scavengers That Collect and Bundle Bacteria." Cell 171 (6): 1368-1382.e23.

https://doi.org/10.1016/j.cell.2017.11.001.

Geiger, Benjamin, Alexander Bershadsky, Roumen Pankov, and Kenneth M. Yamada. 2001.

"Transmembrane Extracellular Matrix-Cytoskeleton Crosstalk." Nature Reviews

Molecular Cell Biology 2 (11): 793-805. https://doi.org/10.1038/35099066. 
Hansen, Caroline E, Yongzhi Qiu, Owen J.T. McCarty, and Wilbur A Lam. 2018. "Platelet Mechanotransduction." Annual Review of Biomedical Engineering 20 (1): 253-75. https://doi.org/10.1146/annurev-bioeng-062117-121215.

Hielscher, Abigail, Kim Ellis, Connie Qiu, Josh Porterfield, and Sharon Gerecht. 2016.

"Fibronectin Deposition Participates in Extracellular Matrix Assembly and Vascular Morphogenesis." PLoS ONE 11 (1): 1-27.

https://doi.org/10.1371/journal.pone.0147600.

Hocking, D C, R K Smith, and P J McKeown-Longo. 1996. "A Novel Role for the Integrin-

Binding III-10 Module in Fibronectin Matrix Assembly." The Journal of Cell Biology 133

(2): 431-44. https://doi.org/10.1083/jcb.133.2.431.

Huang, Derek L., Nicolas A. Bax, Craig D. Buckley, William I. Weis, and Alexander R. Dunn. 2017. "Vinculin Forms a Directionally Asymmetric Catch Bond with F-Actin." Science 357 (6352): 703-6. https://doi.org/10.1126/science.aan2556.

Humphries, Jonathan D., Adam Byron, Mark D. Bass, Sue E. Craig, John W. Pinney, David Knight, and Martin J. Humphries. 2009. "Proteomic Analysis of Integrin-Associated Complexes Identifies RCC2 as a Dual Regulator of Rac1 and Arf6." Science Signaling 2 (87): 1-11. https://doi.org/10.1126/scisignal.2000396.

Katz, Ben-Zion, Eli Zamir, Alexander Bershadsky, Zvi Kam, Kenneth M Yamada, and Benjamin Geiger. 2000. "Physical State of the Extracellular Matrix Regulates the Structure and Molecular Composition of Cell-Matrix Adhesions." Edited by Richard Hynes. Molecular Biology of the Cell 11 (3): 1047-60.

https://doi.org/10.1091/mbc.11.3.1047.

Kechagia, Jenny Z., Johanna Ivaska, and Pere Roca-Cusachs. 2019. "Integrins as

Biomechanical Sensors of the Microenvironment." Nature Reviews Molecular Cell Biology 20 (8): 457-73. https://doi.org/10.1038/s41580-019-0134-2.

Kubow, Kristopher E., Radmila Vukmirovic, Lin Zhe, Enrico Klotzsch, Michael L. Smith, Delphine Gourdon, Sheila Luna, and Viola Vogel. 2015. "Mechanical Forces Regulate the Interactions of Fibronectin and Collagen I in Extracellular Matrix." Nature Communications 6 (1): 8026. https://doi.org/10.1038/ncomms9026.

Lam, Wilbur A, Ovijit Chaudhuri, Ailey Crow, Kevin D Webster, Tai-De Li, Ashley Kita, James Huang, and Daniel A Fletcher. 2011. "Mechanics and Contraction Dynamics of Single Platelets and Implications for Clot Stiffening." Nature Materials 10 (1): 61-66. https://doi.org/10.1038/nmat2903.

Law, Debbie A., Lisa Nannizzi-Alaimo, and David R. Phillips. 1996. "Outside-in Integrin Signal Transduction." Journal of Biological Chemistry 271 (18): 10811-15. https://doi.org/10.1074/jbc.271.18.10811.

Legate, Kyle R., and R. Fassler. 2009. "Mechanisms That Regulate Adaptor Binding to - 
Integrin Cytoplasmic Tails." Journal of Cell Science 122 (2): 187-98.

https://doi.org/10.1242/jcs.041624.

Leiss, Michael, Karsten Beckmann, Amparo Girós, Mercedes Costell, and Reinhard Fässler. 2008. "The Role of Integrin Binding Sites in Fibronectin Matrix Assembly in Vivo."

Current Opinion in Cell Biology 20 (5): 502-7.

https://doi.org/10.1016/j.ceb.2008.06.001.

Li, Shaohua, Caroline Van Den Diepstraten, Sudhir J. D'souza, Bosco M.C. Chan, and J

Geoffrey Pickering. 2003. "Vascular Smooth Muscle Cells Orchestrate the Assembly of Type I Collagen via A2 $\beta 1$ Integrin, RhoA, and Fibronectin Polymerization." The American Journal of Pathology 163 (3): 1045-56. https://doi.org/10.1016/S00029440(10)63464-5.

Lickert, Sebastian, Simona Sorrentino, Jan-Dirk Studt, Ohad Medalia, Viola Vogel, and Ingmar Schoen. 2018. "Morphometric Analysis of Spread Platelets Identifies Integrin Allbß3-Specific Contractile Phenotype." Scientific Reports 8 (1): 5428.

https://doi.org/10.1038/s41598-018-23684-w.

Limouze, John, Aaron F. Straight, Timothy Mitchison, and James R. Sellers. 2004.

"Specificity of Blebbistatin, an Inhibitor of Myosin II." Journal of Muscle Research and Cell Motility 25 (4-5): 337-41. https://doi.org/10.1007/s10974-004-6060-7.

Liu, Jaron, Yilin Wang, Wah Ing Goh, Honzhen Goh, Michelle A Baird, Svenja Ruehland,

Shijia Teo, et al. 2015. "Talin Determines the Nanoscale Architecture of Focal

Adhesions." Proceedings of the National Academy of Sciences 112 (35): E4864-73.

https://doi.org/10.1073/pnas.1512025112.

Lu, Jiaoyang, Andrew D. Doyle, Yoshinari Shinsato, Shaohe Wang, Molly A. Bodendorfer, Minhua Zheng, and Kenneth M. Yamada. 2020. "Basement Membrane Regulates Fibronectin Organization Using Sliding Focal Adhesions Driven by a Contractile Winch." Developmental Cell 52 (5): 631-646.e4. https://doi.org/10.1016/j.devcel.2020.01.007. Malinin, Nikolay L., Li Zhang, Jeongsuk Choi, Alieta Ciocea, Olga Razorenova, Yan-Qing Ma, Eugene A. Podrez, et al. 2009. "A Point Mutation in KINDLIN3 Ablates Activation of Three Integrin Subfamilies in Humans." Nature Medicine 15 (3): 313-18.

https://doi.org/10.1038/nm.1917.

Margadant, Felix, Li Li Chew, Xian Hu, Hanry Yu, Neil Bate, Xian Zhang, and Michael Sheetz. 2011. "Mechanotransduction In Vivo by Repeated Talin Stretch-Relaxation Events Depends upon Vinculin." Edited by Joan Siefert Brugge. PLoS Biology 9 (12): e1001223. https://doi.org/10.1371/journal.pbio.1001223.

Meijden, Paola E J van der, and Johan W M Heemskerk. 2019. "Platelet Biology and Functions: New Concepts and Clinical Perspectives." Nature Reviews Cardiology 16 (3): 166-79. https://doi.org/10.1038/s41569-018-0110-0. 
Myers, David R., Yongzhi Qiu, Meredith E. Fay, Michael Tennenbaum, Daniel Chester, Jonas Cuadrado, Yumiko Sakurai, et al. 2017. "Single-Platelet Nanomechanics Measured by High-Throughput Cytometry." Nature Materials 16 (2): 230-35. https://doi.org/10.1038/nmat4772.

Nechipurenko, Dmitry Y., Nicolas Receveur, Alena O. Yakimenko, Taisiya O. Shepelyuk, Alexandra A. Yakusheva, Roman R. Kerimov, Sergei I. Obydennyy, et al. 2019. "Clot Contraction Drives the Translocation of Procoagulant Platelets to Thrombus Surface." Arteriosclerosis, Thrombosis, and Vascular Biology 39 (1): 37-47. https://doi.org/10.1161/ATVBAHA.118.311390.

Nurden, Paquita, and Alan T. Nurden. 2008. "Congenital Disorders Associated with Platelet Dysfunctions." Thrombosis and Haemostasis 99 (02): 253-63.

https://doi.org/10.1160/TH07-09-0568.

Olorundare, Olufunke E., Olivier Peyruchaud, Ralph M. Albrecht, and Deane F. Mosher. 2001. "Assembly of a Fibronectin Matrix by Adherent Platelets Stimulated by Lysophosphatidic Acid and Other Agonists." Blood 98 (1): 117-24. https://doi.org/10.1182/blood.V98.1.117.

Ono, Akiko, Erik Westein, Sarah Hsiao, Warwick S. Nesbitt, Justin R. Hamilton, Simone M. Schoenwaelder, and Shaun P. Jackson. 2008. "Identification of a Fibrin-Independent Platelet Contractile Mechanism Regulating Primary Hemostasis and Thrombus Growth." Blood 112 (1): 90-99. https://doi.org/10.1182/blood-2007-12-127001.

Rodrigues, Melanie, Nina Kosaric, Clark A. Bonham, and Geoffrey C. Gurtner. 2019.

"Wound Healing: A Cellular Perspective." Physiological Reviews 99 (1): 665-706. https://doi.org/10.1152/physrev.00067.2017.

Sabrkhany, Siamack, Marijke J.E. Kuijpers, Jaco C. Knol, Steven W.M. Olde Damink, AnneMarie C. Dingemans, Henk M. Verheul, Sander R. Piersma, et al. 2018. "Exploration of the Platelet Proteome in Patients with Early-Stage Cancer." Journal of Proteomics 177 (April): 65-74. https://doi.org/10.1016/j.jprot.2018.02.011.

Schoen, Ingmar, Wei Hu, Enrico Klotzsch, and Viola Vogel. 2010. "Probing Cellular Traction Forces by Micropillar Arrays: Contribution of Substrate Warping to Pillar Deflection." Nano Letters 10 (5): 1823-30. https://doi.org/10.1021/nl100533c.

Schoenwaelder, Simone M., Yuping Yuan, Emma C. Josefsson, Michael J. White, Yu Yao, Kylie D. Mason, Lorraine A. O’Reilly, et al. 2009. “Two Distinct Pathways Regulate Platelet Phosphatidylserine Exposure and Procoagulant Function." Blood 114 (3): 66366. https://doi.org/10.1182/blood-2009-01-200345.

Singh, Purva, Cara Carraher, and Jean E Schwarzbauer. 2010. "Assembly of Fibronectin Extracellular Matrix." Annual Review of Cell and Developmental Biology 26 (1): 397419. https://doi.org/10.1146/annurev-cellbio-100109-104020. 
Solari, Fiorella A., Nadine J.A. Mattheij, Julia M. Burkhart, Frauke Swieringa, Peter W. Collins, Judith M.E.M. Cosemans, Albert Sickmann, Johan W.M. Heemskerk, and Rene P. Zahedi. 2016. "Combined Quantification of the Global Proteome, Phosphoproteome, and Proteolytic Cleavage to Characterize Altered Platelet Functions in the Human Scott Syndrome." Molecular and Cellular Proteomics 15 (10): 3154-69. https://doi.org/10.1074/mcp.M116.060368.

Strohmeyer, Nico, Mitasha Bharadwaj, Mercedes Costell, Reinhard Fässler, and Daniel J. Müller. 2017. "Fibronectin-Bound A5 $\beta 1$ Integrins Sense Load and Signal to Reinforce Adhesion in Less than a Second." Nature Materials 16 (12): 1262-70. https://doi.org/10.1038/nmat5023.

Sun, L., J. R. Gorospe, E. P. Hoffman, and A. K. Rao. 2007. "Decreased Platelet Expression of Myosin Regulatory Light Chain Polypeptide (MYL9) and Other Genes with Platelet Dysfunction and CBFA2/RUNX1 Mutation: Insights from Platelet Expression Profiling." Journal of Thrombosis and Haemostasis 5 (1): 146-54. https://doi.org/10.1111/j.15387836.2006.02271.x.

Ting, Lucas H., Shirin Feghhi, Nikita Taparia, Annie O. Smith, Ari Karchin, Esther Lim, Alex St John, et al. 2019. "Contractile Forces in Platelet Aggregates under Microfluidic Shear Gradients Reflect Platelet Inhibition and Bleeding Risk." Nature Communications 10 (1): 1204. https://doi.org/10.1038/s41467-019-09150-9.

Tomaiuolo, Maurizio, Chelsea N. Matzko, Izmarie Poventud-Fuentes, John W. Weisel, Lawrence F. Brass, and Timothy J. Stalker. 2019. "Interrelationships between Structure and Function during the Hemostatic Response to Injury." Proceedings of the National Academy of Sciences 116 (6): 2243-52. https://doi.org/10.1073/pnas.1813642116.

Wang, Yiming, Adili Reheman, Christopher M Spring, Jalil Kalantari, Alexandra H Marshall, Alisa S Wolberg, Peter L Gross, et al. 2014. "Plasma Fibronectin Supports Hemostasis and Regulates Thrombosis." Journal of Clinical Investigation 124 (10): 4281-93. https://doi.org/10.1172/JCl74630.

Wu, C. 1997. "Roles of Integrins in Fibronectin Matrix Assembly." Histology and Histopathology 12 (1): 233-40.

Zamir, Eli, Menachem Katz, Yehudit Posen, Noam Erez, Kenneth M Yamada, Ben-Zion Katz, Shin Lin, et al. 2000. "Dynamics and Segregation of Cell-Matrix Adhesions in Cultured Fibroblasts." Nature Cell Biology 2 (4): 191-96.

https://doi.org/10.1038/35008607.

Zeiler, Marlis, Markus Moser, and Matthias Mann. 2014. "Copy Number Analysis of the Murine Platelet Proteome Spanning the Complete Abundance Range." Molecular \& Cellular Proteomics 13 (12): 3435-45. https://doi.org/10.1074/mcp.M114.038513. Zhang, Yun, Yongzhi Qiu, Aaron T. Blanchard, Yuan Chang, Josh M. Brockman, Victor Pui- 
Yan Ma, Wilbur A. Lam, and Khalid Salaita. 2018. "Platelet Integrins Exhibit Anisotropic

Mechanosensing and Harness Piconewton Forces to Mediate Platelet Aggregation."

Proceedings of the National Academy of Sciences 115 (2): 325-30.

https://doi.org/10.1073/pnas.1710828115.

Zhu, Jianghai Jieqing, Won-seok W.-S. Choi, Joshua G. McCoy, Ana Negri, Jianghai Jieqing

Zhu, Sarasija Naini, Jihong Li, et al. 2012. "Structure-Guided Design of a High-Affinity

Platelet Integrin Allbß3 Receptor Antagonist That Disrupts Mg2+ Binding to the

MIDAS." Science Translational Medicine 4 (125): 125ra32-125ra32.

https://doi.org/10.1126/scitranslmed.3003576.

Zimman, Alejandro, Bjoern Titz, Evangelia Komisopoulou, Sudipta Biswas, Thomas G.

Graeber, and Eugene A. Podrez. 2014. "Phosphoproteomic Analysis of Platelets

Activated by Pro-Thrombotic Oxidized Phospholipids and Thrombin." Edited by Jens

Schlossmann. PLoS ONE 9 (1): e84488. https://doi.org/10.1371/journal.pone.0084488. 